\title{
Three-dimensional cardiac computational modelling: methods, features and applications
}

\author{
Alejandro Lopez-Perez ${ }^{1 *}$, Rafael Sebastian ${ }^{2}$ and Jose M Ferrero ${ }^{1}$
}

\author{
* Correspondence: \\ alopez@gbio.i3bh.es \\ ${ }^{1}$ Centre for Research and Innovation \\ in Bioengineering (Ci2B), Universitat \\ Politècnica de València, València, \\ Spain \\ Full list of author information is \\ available at the end of the article
}

\begin{abstract}
The combination of computational models and biophysical simulations can help to interpret an array of experimental data and contribute to the understanding, diagnosis and treatment of complex diseases such as cardiac arrhythmias. For this reason, three-dimensional (3D) cardiac computational modelling is currently a rising field of research. The advance of medical imaging technology over the last decades has allowed the evolution from generic to patient-specific 3D cardiac models that faithfully represent the anatomy and different cardiac features of a given alive subject. Here we analyse sixty representative 3D cardiac computational models developed and published during the last fifty years, describing their information sources, features, development methods and online availability. This paper also reviews the necessary components to build a 3D computational model of the heart aimed at biophysical simulation, paying especial attention to cardiac electrophysiology (EP), and the existing approaches to incorporate those components. We assess the challenges associated to the different steps of the building process, from the processing of raw clinical or biological data to the final application, including image segmentation, inclusion of substructures and meshing among others. We briefly outline the personalisation approaches that are currently available in 3D cardiac computational modelling. Finally, we present examples of several specific applications, mainly related to cardiac EP simulation and model-based image analysis, showing the potential usefulness of 3D cardiac computational modelling into clinical environments as a tool to aid in the prevention, diagnosis and treatment of cardiac diseases.
\end{abstract}

Keywords: Cardiac modelling, Three-dimensional (3D) modelling, Computational modelling, Fibre orientation, Cardiac conduction system (CCS), Cardiac image segmentation, Biophysical simulation, Personalisation, Patient-specific modelling

\section{Introduction}

Some decades ago, three-dimensional (3D) cardiac computational models were only used for very simple computational simulations of cardiac electrophysiology (EP) or cardiac mechanics analysis. Nowadays, 3D cardiac models are becoming increasingly complex and are currently used in other areas such as cardiac image segmentation, statistical modelling of cardiac anatomy, patient risk stratification or surgical planning. These models are starting to be used in clinical environments for 3D image analysis or therapy guidance in procedures such as radiofrequency ablation (RFA). Due to the intensive research in this field and the evolution of computing resources, the introduction of 3D advanced computational simulations of cardiac EP and/or mechanics and

(c) 2015 Lopez-Perez et al.; licensee BioMed Central. This is an Open Access article distributed under the terms of the Creative Commons Attribution License (http://creativecommons.org/licenses/by/4.0), which permits unrestricted use, distribution, and reproduction in any medium, provided the original work is properly credited. The Creative Commons Public Domain Dedication waiver (http://creativecommons.org/publicdomain/zero/1.0/) applies to the data made available in this article, unless otherwise stated. 
model-based cardiac image analysis in clinical environments are becoming more feasible.

This paper presents a review of the methods used to construct 3D cardiac computational models since their earliest developments (about fifty years ago) until today, and discusses their advantages and applicability to different areas. To carry out our systematic review, sixty representative computational models were taken from the literature and were analysed in order to explore the evolution of the methods used to develop 3D cardiac models over the last fifty years. As a main result, we crafted a wide summary table (see Additional file 1: Table S1) that provides information about the main features of the reviewed 3D cardiac models and the particular methods used to build each of them.

This article is organised as follows. We first discuss the information contained in (Additional file 1: Table S1) and its intended usefulness for the readers. Later, we outline the evolution of $3 \mathrm{D}$ cardiac models from the "early era" to the present days, highlighting the methods used for the computational reconstruction of cardiac anatomy. The next section addresses the different stages of the development process of a 3D cardiac model (3D reconstruction of cardiac anatomy, meshing, etc.) and reviews the available methods to construct a model and to include certain heart features (fibre orientation, cardiac conduction system, ischaemic scars, etc.) in a computational model aimed at biophysical simulation with especial attention to cardiac EP. The following section briefly describes the available personalisation approaches in cardiac computational modelling. Finally, the paper addresses the main applications of 3D cardiac models by presenting examples related to several specific applications, focusing on cardiac EP simulation and model-based image segmentation.

\section{Table of 3D cardiac computational models}

Reviewing the entire literature related to the development of 3D cardiac models would be virtually impossible. For this reason, we chose sixty models from the literature as a representative set suitable to outline the evolution of 3D cardiac computational modelling from its beginning. In order to show this evolution we list them in chronological order in (see Additional file 1: Table S1).

Additional file 1: Table S1, crafted as a main result of this review work, was designed to provide a complete summary about the reviewed models. It shows information about the data source and methods used to develop each of the sixty reviewed 3D cardiac models as well as their main features, final application and online availability, in case the reader is interested in downloading any of them. The information provided by each column of Additional file 1: Table S1 is later addressed in a specific subsection of the Elements of a 3D cardiac computational model section, discussing why certain features or methods are needed or convenient for particular applications. We intend for readers to use Additional file 1: Table S1 as a reference tool along the entire article since it contains examples of models including the different cardiac features addressed or models that were developed using some of the methods mentioned in the text. Therefore, it can be used to find several models sharing a particular purpose or certain feature/ method in which the reader might be especially interested or to compare different models in a quick and straightforward manner. 


\section{Evolution of 3D models of cardiac anatomy}

The first step of the development process of a 3D cardiac model is the computational reconstruction of the anatomy of the heart by generating a 3D cardiac geometry. In this section, a brief survey of the evolution of 3D cardiac models is presented focusing on the methods used to build the computational reconstruction of cardiac anatomy and the achieved level of anatomical detail.

\section{Generic models}

The first developed 3D computational models of cardiac anatomy were simplistic models based on geometric shapes. Most of them only included the left ventricle (LV), represented by two concentric ellipsoids truncated at the base level to roughly approximate the shape of the LV [1-5]. However, this approach is still in use for specific applications in which the anatomical realism is not crucial for the purpose of the model [6,7].

Later, anatomical models were established. They aimed to represent cardiac anatomy in a more realistic fashion but still with a low level of anatomical detail due to the poor quality of the data used to build them. They were usually constructed by manual drawing from histo-anatomical slices [8-11] or from measurements taken on explanted hearts [12,13] or by segmenting pictures of histo-anatomical slices [14-17]. The most representative ones are two bi-ventricular models highly referenced and reused: the rabbit model from University of California San Diego [11] and the canine model from University of Auckland [12]. Their main contribution was the inclusion of realistic fibre orientation obtained from experimental measurements.

The development of computer-aided design (CAD) tools enabled the construction of 3D cardiac models without any direct source of anatomical information [18-21]. Some anatomical details, such as chambers volumes or wall thickness were just taken from the literature in order to virtually generate the geometry of the model.

3D atrial models began proliferating later than ventricular ones for several reasons, such as the higher lethality of ventricular disorders or the challenges associated to its $3 \mathrm{D}$ reconstruction due to the high complexity and inter-subject variability of atrial anatomy. Nevertheless, all kinds of model described above are present among reviewed 3D atrial models: geometric models [22], CAD models [19,21] and anatomical models from histo-anatomical slices [23-25].

\section{Medical image-based models}

The evolution of medical imaging technology gave the possibility of building realistic 3D cardiac models from either in-vivo or ex-vivo images, as demonstrated by early works [26] and [27], respectively. Medical image-based 3D cardiac models have proliferated over the last 15 years due to the advance and consolidation of techniques such as magnetic resonance imaging (MRI) [28-34] and computed tomography (CT) [35,36], leading to the rise of $3 \mathrm{D}$ cardiac computational modelling. As will be discussed below, the development of new imaging modalities capable of providing structural and functional information of cardiac tissue was also a major breakthrough in 3D cardiac computational modelling.

The increasing availability of in-vivo cardiac images together with the rising trend towards personalised medicine resulted in the definition of patient-specific models. They 
model the cardiac anatomy of a specific human subject from in-vivo images, usually MRI [37-39] or CT [40,41]. Figure 1 shows a patient-specific bi-ventricular model built from in-vivo MRI [39]. Building this kind of model requires imaging techniques synchronised with the ECG and breathing in order to overcome the noise and motion artefacts due to the cardiac cycle and breathing movements. This has also enabled building dynamic models that include the intra-subject anatomical variations of the heart due to the cardiac cycle $[37,38]$.

Cardiac atlases also emerged thanks to the increasing availability of in-vivo images. They are assembled by averaging several 3D cardiac image datasets from a population of subjects, thus generating a mean 3D cardiac image or shape (for further details about cardiac atlases see Cardiac image segmentation section). For instance, the cardiac atlas developed in [42] was constructed from 14 manually segmented cine-MRI images and in [43] in-vivo multislice-CTs (MS-CT) from 100 subjects were used.

There are a few highly-detailed bi-ventricular models built from very high resolution ex-vivo MRI datasets ( $25 \mu \mathrm{m}$ per slice) from small mammalian hearts, which show an outstanding level of anatomical detail including papillary muscles and endocardial trabeculations. Some of them even take into account detailed information at tissue level provided by histological slices with specific staining [44,45]. Figure 2 shows an example of a highly-detailed rabbit bi-ventricular model [46].

\section{Elements of a 3D cardiac computational model}

In addition to the 3D geometry representing part of the cardiac anatomy, every $3 \mathrm{D}$ cardiac computational model may also require other elements, such as the structure of the cardiac tissue, biophysical models of the heart (EP and/or mechanical), pathologies that affect the myocardium, etc. In this section we review the data sources and computational methods used to include those elements into a model, also specifying which of them are necessary depending on the final purpose of the model. Figure 3 shows a flowchart depicting the full development pipeline of a 3D cardiac computational model aimed at biophysical simulation, showing the main stages of the building process and the relationships between them. These steps will be addressed in the following sections,

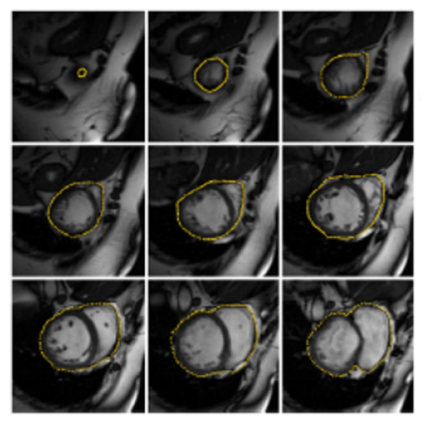

(a)

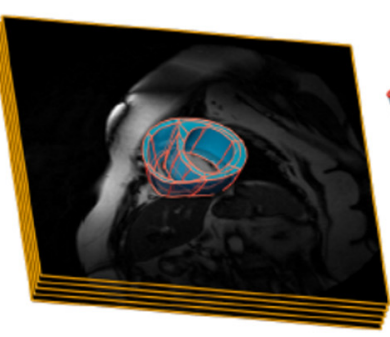

(b)

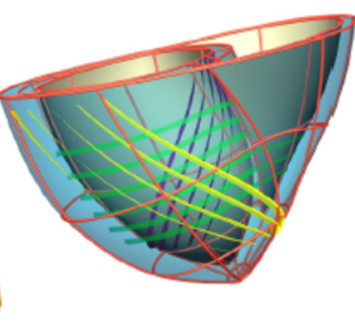

(c)

Figure 1 Patient-specific bi-ventricular model. (a) In-vivo cardiac MRI slices showing manually segmented epicardial contour. (b) 3D cardiac model overlaid on the MRI stack. (c) Finite-element mesh with tri-cubic Hermite elements showing the main direction of fibre orientation at epicardium (yellow), midwall (green) and endocardium (purple). Reproduced with permission from [39] 

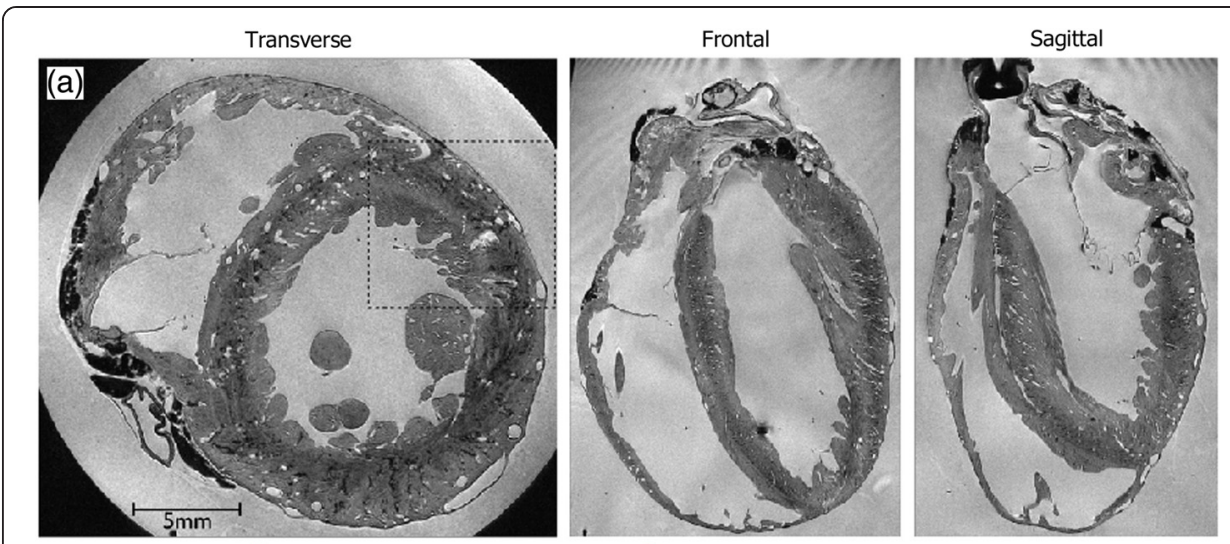

(b)

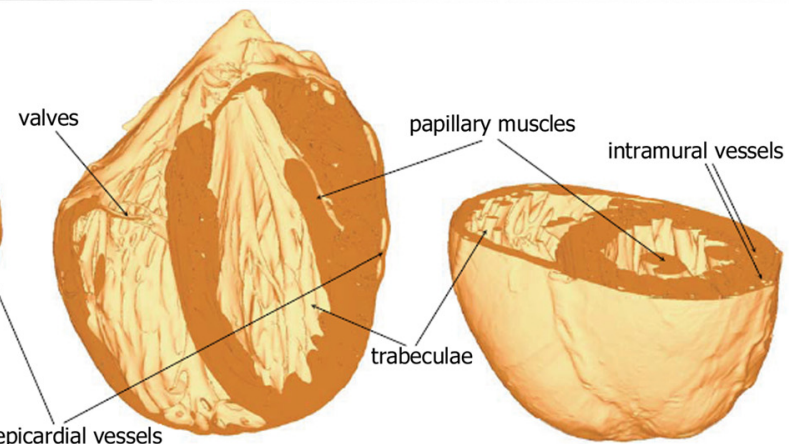

(c)
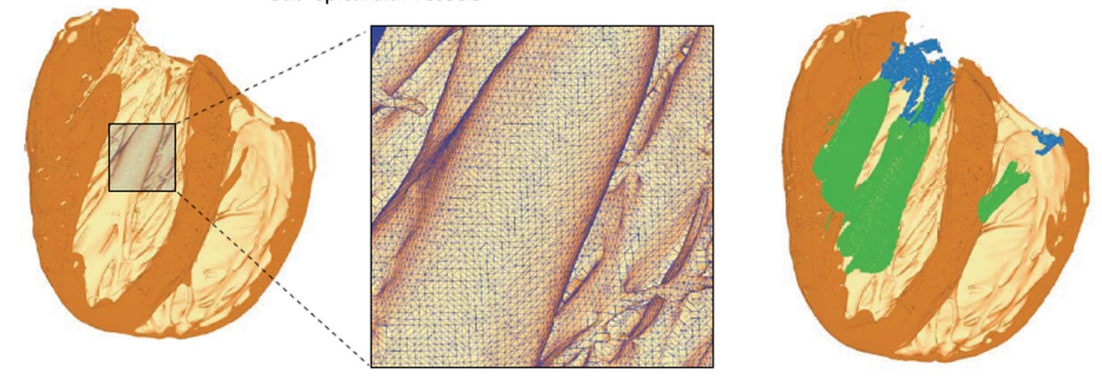

Figure 2 Highly-detailed rabbit bi-ventricular model. (a) Very high resolution ex-vivo MRI. (b) 3D rendering of the model showing a high level of anatomical detail. (c) Detail of tetrahedral finite-element mesh showing the papillary muscles (green) and chordae tendineae (blue). Adapted with permission from [46].

mainly focusing on cardiac EP simulation and providing an extended diagram specific to each step.

\section{Geometry}

As shown in Figure 3, the generation of a 3D cardiac geometry, usually represented by a 3D surface mesh, is the very first step of the construction process of a 3D cardiac model. The geometry of the heart is a key feature that must be represented by $3 \mathrm{D}$ cardiac models accurately and realistically. In general, the geometry of a 3D model represents one or several cardiac chambers (LV, bi-ventricular, atrial or whole-heart models) and can also include other details such as the great cardiac vessels including outflow and/or inflow tracts $[17,47,48]$, the fibrous annulus of atrioventricular valves $[49,50]$, part of the coronary tree, or some endocardial details such as papillary muscles and trabeculae carneae for ventricles or crista terminalis, pectinate muscles and fossa ovalis 


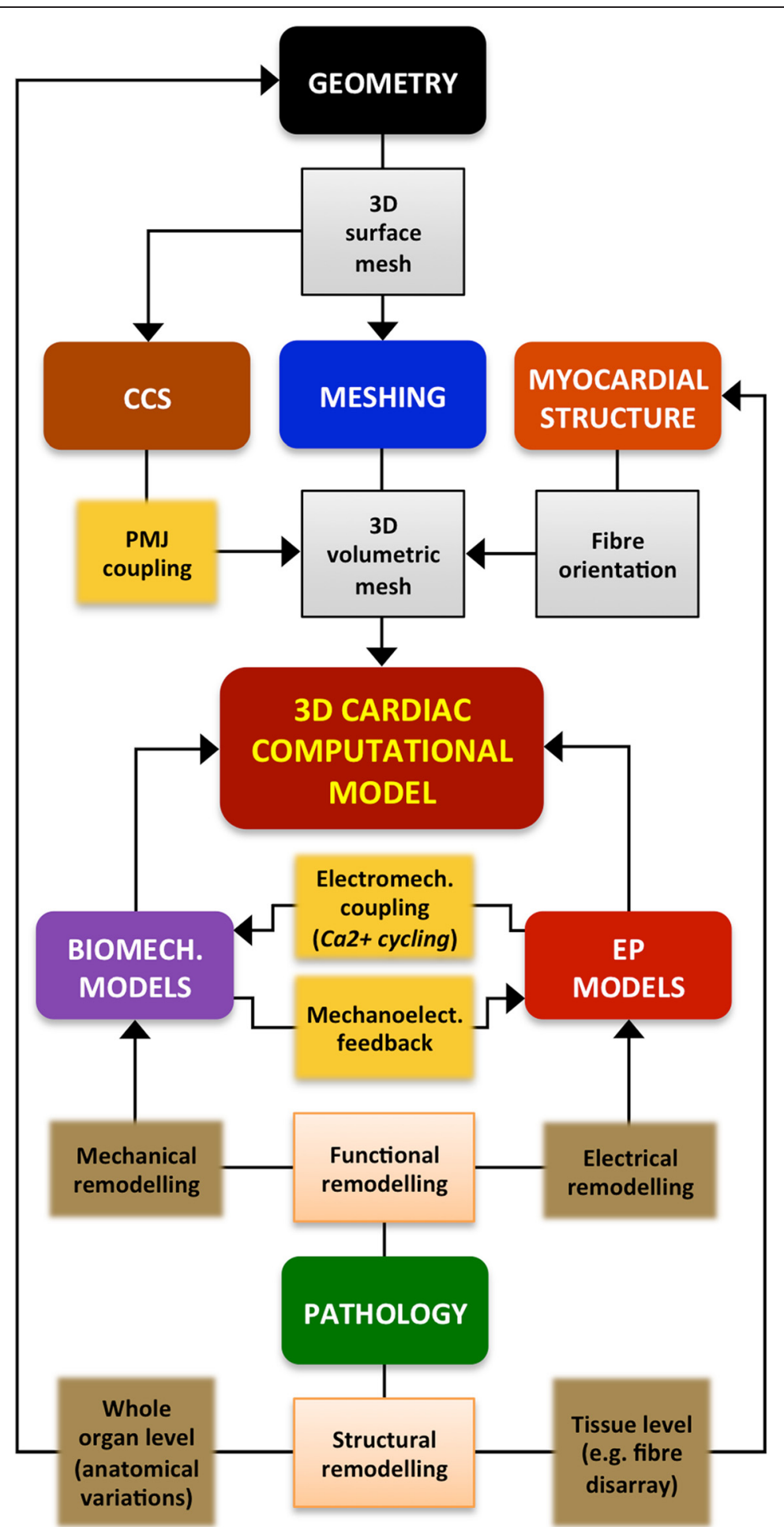

Figure 3 Full pipeline to build a 3D cardiac computational model aimed at biophysical simulation. Summarised flowchart showing the main stages of the construction of a 3D cardiac model aimed at biophysical simulation: 3D cardiac geometry generation, meshing, CCS generation, myocardial structure generation, biophysical modelling (cardiac EP and biomechanics) and cardiac pathology modelling. Lines and arrows depict the relationships between the different stages by means of partial results (grey boxes) and coupling steps (yellow boxes). For pathology modelling, the diagram shows the different types (orange boxes) and subtypes (brown boxes) of cardiac pathology that can be included in a cardiac computational model and the stage in which each type of pathology must be taken into account. 
for atria $[21,24,25]$. However, it is important to note that the completeness and the anatomical realism and accuracy required by a particular 3D cardiac model will strongly depend on its final application. In [46] it was concluded that structurally simplified models (without endocardial details or vessels) are well suited for a large range of 3D cardiac modelling applications aimed at EP simulation, although the presence of trabeculae provides shortcut paths for excitation causing regional differences in electrical activation patterns after pacing compared to anatomically non-detailed models.

The level of anatomical detail achieved by a given model also depends strongly on the source of anatomical information and the methodology used to build it, as shown in Figure 4. Geometric or CAD models, whose geometry shows a coarse representation of cardiac anatomy, are built from population-based data just taking into account some measurements of cardiac chambers volume or wall thickness $[4,18]$. They are normally used when no direct source of anatomical information is available or when the simplicity of the geometry is preferred to the anatomical realism for the purpose of the model $[6,7,22]$. Histo-anatomical slices can provide highly detailed anatomical [25]

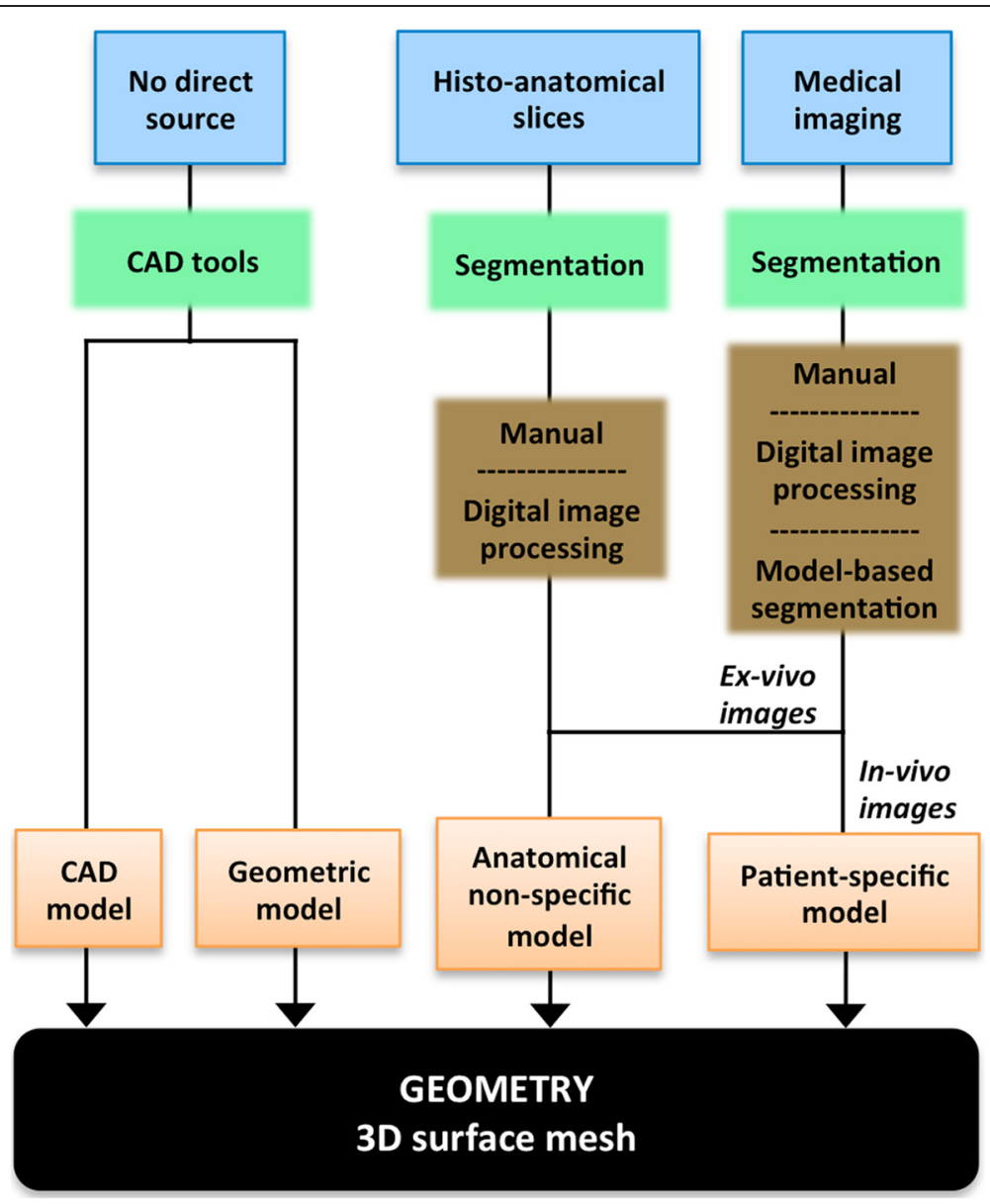

Figure 4 3D cardiac geometry generation stage of the development process of a 3D cardiac computational model. Diagram depicting the main alternatives to generate the 3D surface mesh that represents the cardiac geometry, showing the sources of anatomical information (blue boxes) and the methods (green boxes) with their possible options (brown boxes) used for this task, as well as the kind of model (orange boxes) obtained by each method. 
and also histological information $[44,45]$. However, there is usually a large gap between adjacent slices what leads to the loss of great amount of information out of plane $[9,10,14]$, although it can be mitigated by means of interpolation techniques.

Medical image-based models can include patient-specific details obtained from clinical imaging data and/or population-based properties collected from ex-vivo datasets (see Figure 4). Clinical imaging protocols usually provide sparse datasets with large gaps between slices, as in the case of most MRI modalities (e.g. [38,49,51]), what often leads to the use of interpolation schemes. Nonetheless, due to the advance of the imaging techniques this approach can require the segmentation of large stacks of tomographic images, especially for high-resolution ex-vivo datasets (e.g. [32,35,36,46]) or cardiac atlases whose construction involves segmenting numerous in-vivo datasets (e.g. [43,52]). Manual segmentation requires expertise and is very time consuming, while automatic segmentation of cardiac images is still challenging, especially for in-vivo datasets. Despite this, clinical imaging techniques (mainly MRI and CT) are today the source of anatomical information most commonly used to generate the geometry of 3D cardiac models.

Ex-vivo cardiac images can provide much higher spatial resolution than in-vivo datasets for several reasons: absence of motion artefacts, removal of surrounding tissue before the scan and lack of the limitations imposed by alive subjects (either human or non-human) regarding the acquisition time and the ionizing radiation dose (in the case of CT modalities). It allows detailed reconstructions of cardiac geometry, including structures very difficult to observe in in-vivo images such as Bachmann's bundle or pectinate muscles in the atria and endocardial trabeculations in the ventricles [31,45] or leaflets of the cardiac valves and the chordae tendineae [46]. Recently, ex-vivo micro-CT with iodine staining has allowed reconstructing structures such as the atrioventricular node and atrial preferential conducting bundles [36]. Among the reviewed works, the segmentation of ex-vivo images was usually performed by bidimensional (2D) semi-automatic approaches (slice by slice) by combining classical image processing methods such as region growing [31,35], snakes $[28,30]$ or level sets $[32,34]$. However, manual correction was needed in most cases after the automatic segmentation process $[30,31,34,35]$. For those models based on very high resolution exvivo MRI, 2D semi-automatic segmentation was also applied but with a lower level of manual interaction, e.g. using thresholding and morphological operators [44] or complex pipelines based on level sets $[45,46,53]$.

In-vivo images can provide both anatomical and temporal patient-specific information, thus enabling the characterisation of cardiac motion [52,54]. The reviewed patient-specific models based on in-vivo MRI were mostly assembled by manual segmentation [37,39]. Images provided by certain MRI modalities, such as cine-MRI, can be segmented by 2D automatic approaches combining morphological operators and snakes [38]. 2D semi-automatic approaches based on snakes/level sets [40] and even 3D automatic methods [41] were applied to in-vivo MS-CT. Some cardiac atlases were also assembled from manually segmented MRI $[51,55]$. Nevertheless, to facilitate the segmentation of large amount of datasets, more complex approaches have been applied to assemble cardiac atlases: fitting of a deformable model based on geometrical shapes followed by manual correction [56], adaption of an initial mesh by piecewise affine 
transformation [47] or non-rigid registration with a previously manually segmented image $[43,52,54]$.

In conclusion, high-resolution ex-vivo datasets enable much more detailed reconstructions of cardiac anatomy than in-vivo ones. However, in addition to the explantation of the heart, the organ must undergo a whole process of tissue preparation (fixation, chambers filling, etc.) before the acquisition of ex-vivo cardiac datasets, either ex-vivo images or histological slices. This process could alter several features of cardiac structures, such as shape, size, volume, etc., especially in the case of histological sections due to the deformation caused by the slicing process [57-59]. Therefore, even though it is undoubtedly a good approximation, today it still remains unclear to what extent an ex-vivo derived geometry is relevant to the in-vivo function of the heart, as posed in [26]. To our knowledge, there is no literature addressing this issue thoroughly, so it is something to take into account when a 3D cardiac model is used to carry out computational simulation studies with potential clinical relevance.

Cardiac models can also include the coronary tree, which is often virtually generated from the anatomical knowledge, manually segmented from pictures of histo-anatomical slices [17] or fitted from a previous model [56]. The full coronary tree can be segmented from very high resolution ex-vivo MRI $[44,46,53]$. Using complex segmentation pipelines the main coronary arteries can be reconstructed from in-vivo MRI [37]. However, high-resolution MS-CT has become the modality for in-vivo assessment of the structure of the coronary tree since it allows segmenting part of the patient-specific cardiac vascular network $[43,52]$. There are some applications in which the coronary tree might have a central role in the model, such as cardiac resynchronisation therapy (CRT) where the implanted leads are spatially restricted to the lumen of some specific veins $[60,61]$. Other authors have also studied the role played by blood vessels (e.g. fibre orientation changes around vessels) within the heart in stabilising arrhythmias, reporting changes in wavefront curvature around the blood vessels [62].

\section{Meshing}

Although simple heart models still play an important role for certain applications that focus on mechanistic enquiry, current trends are moving towards patient-specific complex anatomical models. Both simple and detailed anatomical heart models are commonly represented by 3D elements resulting from a meshing process. Figure 5 shows an overview of the most common meshing options for 3D cardiac models. The homogenisation of discrete tissue components and the adoption of advanced spatial discretisation techniques based on the finite-element method (FEM) have enabled the resolution of complex biophysical problems. As shown in Figure 3 and Figure 5, anatomical models are usually represented by discrete 3D surface meshes resulting from the geometry generation stage, which will serve as an input for a volumetric mesh generator software (e.g. Tetgen, NetGen, Tarantula). The most common alternative to FEM method is based on grid-based meshes, which can operate directly from a segmented image stack to discretise the volume [45] (see Figure 5).

For EP simulations, unstructured volumetric FEM meshes are commonly used consisting of linear elements that are usually tetrahedral [46], hexahedral or a combination of both [63]. The use of hexahedral elements is desirable to decrease the number of degrees of freedom of FEM models, at the cost of a poorer representation of cardiac 


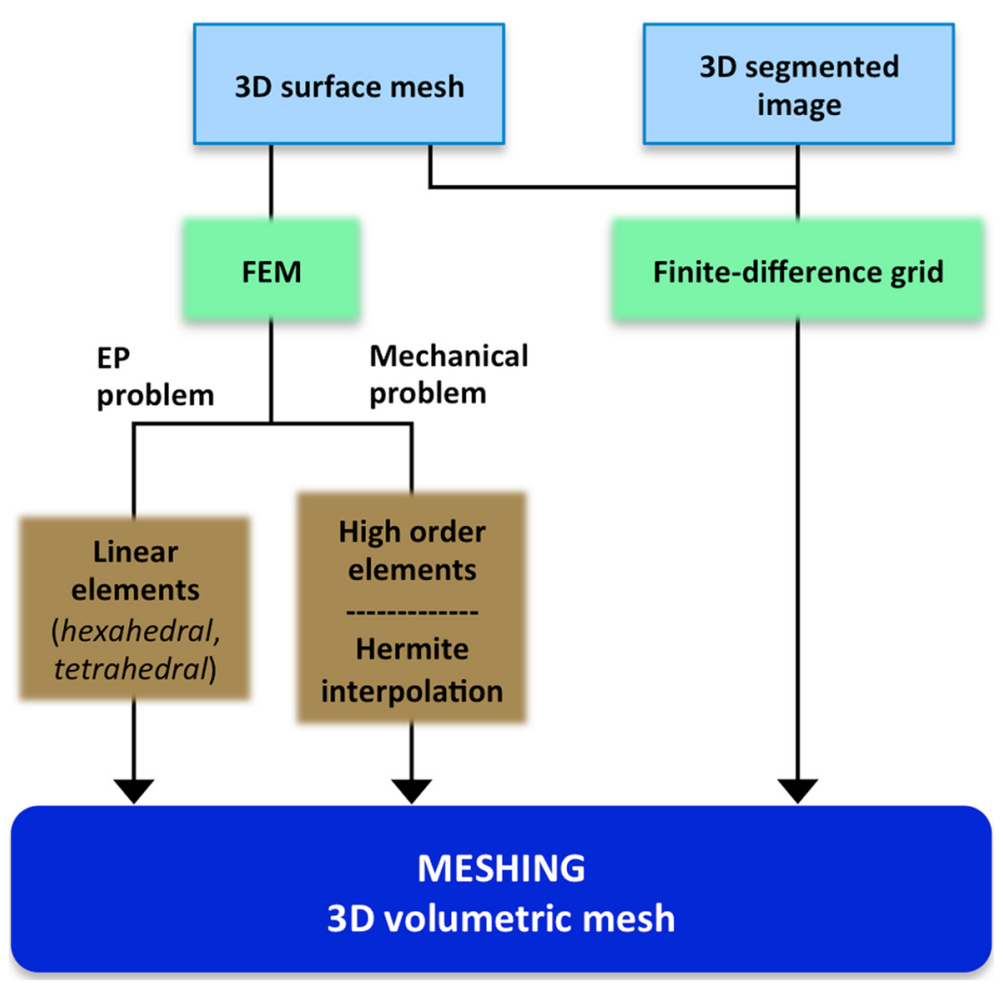

Figure 5 Meshing stage of the development process of a 3D cardiac computational model. Diagram describing the most common methods (green boxes) and options (brown boxes) to build the 3D volumetric mesh of a cardiac model using the 3D surface mesh or the 3D segmented image resulting from the cardiac geometry generation as a starting point for the meshing process.

anatomy [33,64]. Another extended representation of cardiac anatomy uses cubic Hermite elements, which provide a smooth representation of the model geometry that is well-suited to simulate large deformation mechanics [65]. Although that representation fails to faithfully represent the subtle anatomical details present on the heart, it shows a higher numerical accuracy for mechanical simulations than linear interpolation schemes in models based on tetrahedral or hexahedral elements [66]. Indeed, models aimed at electromechanical simulations usually include two coupled FEM volumetric meshes: one based on linear elements to solve the electrical component and one based on higher order elements [6] or Hermite interpolation functions [34] for the mechanical problem.

The equations to be solved on FEM models impose strong restrictions on mesh elements. In addition, the inclusion of fine anatomical structures (Purkinje, trabeculae, vascularisation) to faithfully represent the cardiac anatomy also increases the number of degrees of freedom of a model. Spatial (ds) and temporal discretisation (dt) constraints are imposed when biophysical models are used, which are in the order of $\mathrm{ds}=0.1-0.5 \mathrm{~mm}$ and $\mathrm{dt}=0.05-0.005 \mathrm{~ms}$ [67]. The main reason is the fast upstroke of cellular depolarisation, which produces a step-like wavefront over a small spatial extent [68]. For the case of phenomenological models, such as Eikonal ones, spatial and temporal discretisation is less demanding (order of $\mathrm{ds}=0.5 \mathrm{~mm}, \mathrm{dt}=1 \mathrm{~ms}$ ), resulting in faster computation times. 


\section{Myocardial structure}

Cardiac myocytes are elongated cells arranged in a laminar sheet organisation to form the ventricular myocardium [69,70]. The direction of the longitudinal axis of cardiac myocytes, known as fibre orientation, strongly determines the electrical activation pattern of myocardium since the electrical propagation is 3 to 4 times faster along this axis than in the transversal one [71]. Furthermore, myocardial contraction is characterised by a shrinkage along the longitudinal axis of myocytes, so fibre orientation has also a great influence on the mechanical behaviour of cardiac tissue. Thus, fibre orientation must be included in models aimed at performing realistic EP and/or mechanical computational simulations. Once the 3D volumetric mesh resulting from the meshing stage is built, the fibre orientation may be included in the $3 \mathrm{D}$ model by setting the direction of the longitudinal axis as a property of every volume mesh element (see Figure 3).

Figure 6 shows a schematic summary of the methods most commonly used to obtain the fibre orientation of myocardial tissue. The most usual approach is based on rulebased algorithms that estimate the fibre orientation associated to each element of the volumetric mesh of a model from pre-established patterns [5,6,41,43], most of them derived from Streeter's findings [72]. Fibre orientation can also be obtained from measurements taken on explanted hearts [12,35], by analysing histological sections under microscope [11] or by digital processing (structure tensor method) of volume images assembled from high-resolution pictures of very thin histological slices [25,44].

Diffusion tensor-MRI (DT-MRI), also called diffusion tensor imaging (DTI), is a MRI modality capable of showing the diffusion of water molecules within the biological tissues. For cardiac DTI, it is well known that the direction of the primary eigenvector associated to each voxel of the acquired images matches the longitudinal axis of cardiac

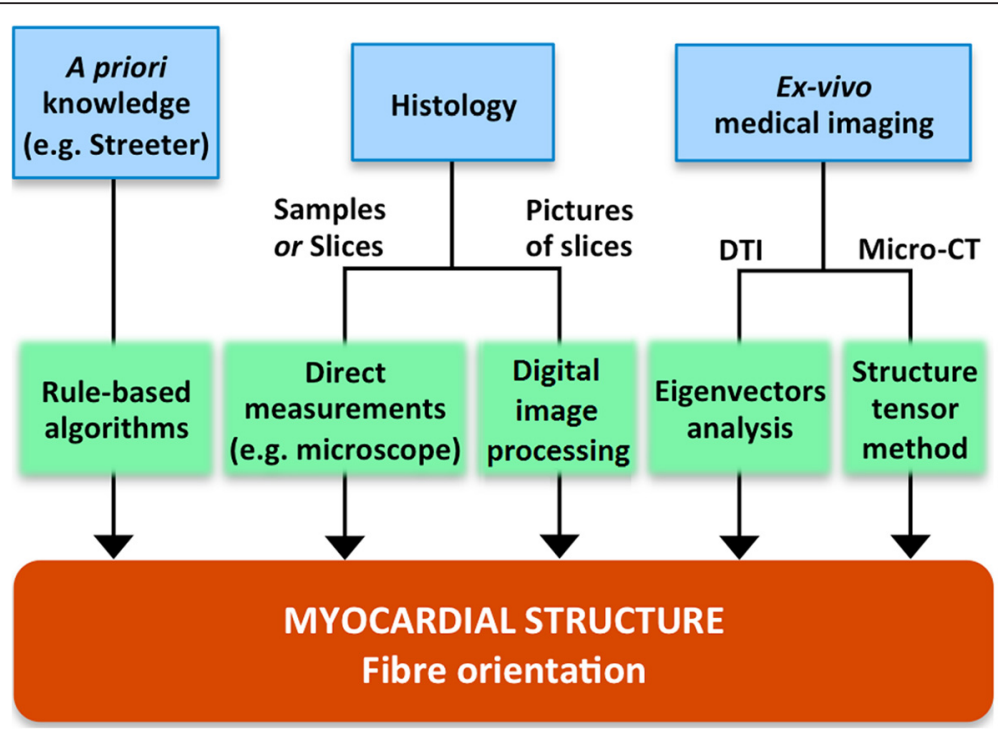

Figure 6 Myocardial structure generation stage of the development process of a 3D cardiac computational model. Diagram showing the main sources of structural information at tissue level (blue boxes) and the methods (green boxes) used to obtain the fibre orientation to be included in a 3D cardiac model. 
myocytes [73-75]. This information can be mapped onto the volumetric mesh of a 3D cardiac model to include fibre orientation [31,34,45,76]. In [77] a statistical atlas that characterises the variability of fibre orientation was constructed using ex-vivo DTI from nine canine hearts. In recent works there have been proposed approaches to estimate the patient-specific fibre orientation of the LV from sparse in-vivo 2D DTI slices [78,79] benefiting from the aforementioned fibre statistical atlas [77]. Ex-vivo cardiac DTI can also provide anatomical information, thus avoiding the need to merge different image modalities to construct a 3D cardiac model including fibre orientation [30,33]. However, due to its high sensitivity to motion artefacts, in-vivo cardiac DTI is not capable of providing the full patient-specific fibre orientation of the whole heart yet. In [80] it was shown that global electrical activation patterns obtained by computational simulation from a model with fibre orientation based on a rule-based linear approach were very similar to those based on DTI for the same geometry, thus demonstrating the robustness of the former method for EP simulation studies. Likewise, in [81] a novel rule-based algorithm was compared to DTI-derived fibre orientation (see Figure 7) reaching similar conclusions. Micro-CT with iodine staining is another image modality recently used to assess the fibre orientation in certain critical regions of atrial tissue by structure tensor method [36]. Nevertheless, in-vivo micro-CT is not feasible because of the needed high dose of ionizing radiation. In conclusion, currently there is no in-vivo technique capable of providing the full patient-specific fibre orientation of the whole heart.

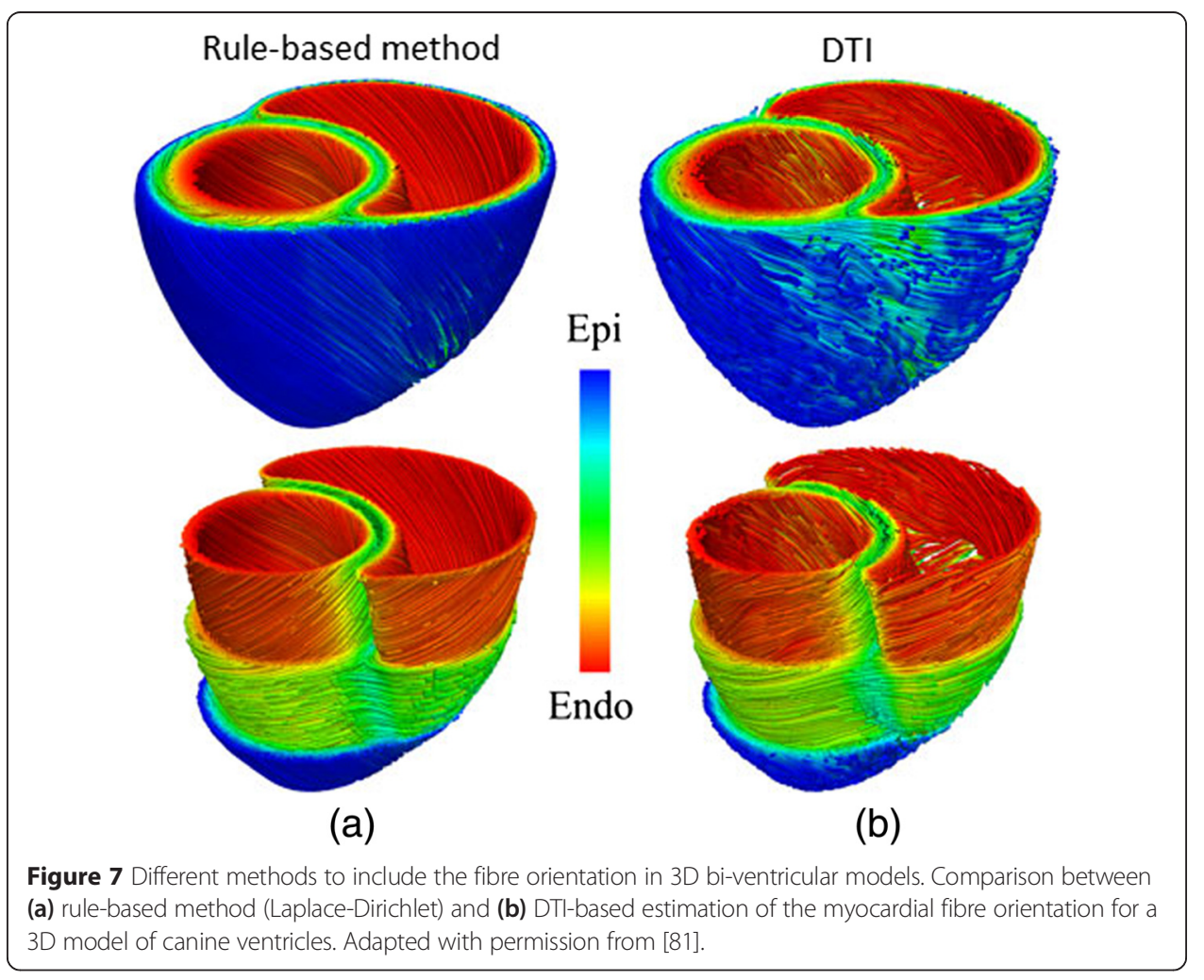




\section{Cardiac conduction system}

The electrical activation of the ventricles is triggered by a heterogeneous complex $3 \mathrm{D}$ structure network that combines subendocardial and free-running fibres forming the so-called cardiac conduction system (CCS). The ventricular portion of the CCS ensures the coordinated activation of the ventricular myocardium to achieve the most efficient pumping activity [82]. The His-Purkinje system is highly specialised for a rapid conduction ( 2-4 m/s) of the action potential (AP). The CCS is isolated from surrounding myocardium by connective tissue sheaths along its path with the exception of the terminal portion, allowing AP to propagate to ventricular working myocardium at the Purkinje-myocardium junctions (PMJs) [83]. The location of these PMJs plays a key role since they are the source points of the activation of the ventricles [82]. A number of EP computational studies have already integrated CCS models to analyse their role in non-physiological scenarios such as during ventricular tachy-arrhythmias $[84,85]$, ventricular pacing [41] or administration of antiarrhythmic drugs [64]. All these studies reproduce Purkinje cell EP using tailored AP models $[86,87]$ that capture their main electrical behaviour. The most recently developed Purkinje EP models include the $\mathrm{Ca} 2+$ subsystem spatial organisation and receptors distribution that are unique to Purkinje cells [88], allowing a more accurate modelling of arrhythmias.

Figure 8 shows the main steps and alternatives to generate a model of the CCS that can be coupled to a 3D cardiac model, as shown in Figure 3. The CCS can be visualised using ex-vivo microscopic images with specific markers such as stains or connexin antibodies, but cannot be reconstructed from in-vivo imaging techniques since its structures are under the level of resolution of current clinical imaging systems. The methods to include the CCS function in computational models are often based on altered endocardial properties that emulate faster activations [89], the setup of a number of early activation points obtained from the literature (e.g. from [90]) or electrical recordings [91], or simplified models that aim to emulate the structure of the CCS network. The latter models can be constructed using different techniques such as manual delineation of CCS on the endocardial surfaces of a 3D cardiac model [41], fractal tree-like representations [92], or algorithms based on the characterisation of the main features of the CCS structure obtained from ex-vivo population data by means of histological studies of animal hearts (rat, rabbit, dog or lamb) [93]. Very high resolution ex-vivo MRI has allowed locating a number of free-running Purkinje fibres by visual inspection [53,94] and recently ex-vivo micro-CT with iodine staining has enabled an image-based semi-automatic reconstruction of the full CCS [95], all in small mammalian hearts.

It is important to highlight that due to the special inhomogeneous coupling between CCS and ventricular working myocardium at PMJs, specific computational models are required for the PMJ coupling (see Figure 3). Those models try to reproduce the propagation delay at PMJs in healthy and pathological conditions, adding transitional regions or resistor elements to couple both cell types [84,96-98]. However, it is still very difficult to determine the location and density of PMJs, and therefore computational studies can provide insights into this important matter in normal and pathological scenarios [85]. Recently, novel methods to estimate the location of PMJs clusters and the structure of the CCS from in-vivo electroanatomical maps (EAMs) have appeared $[99,100]$. 


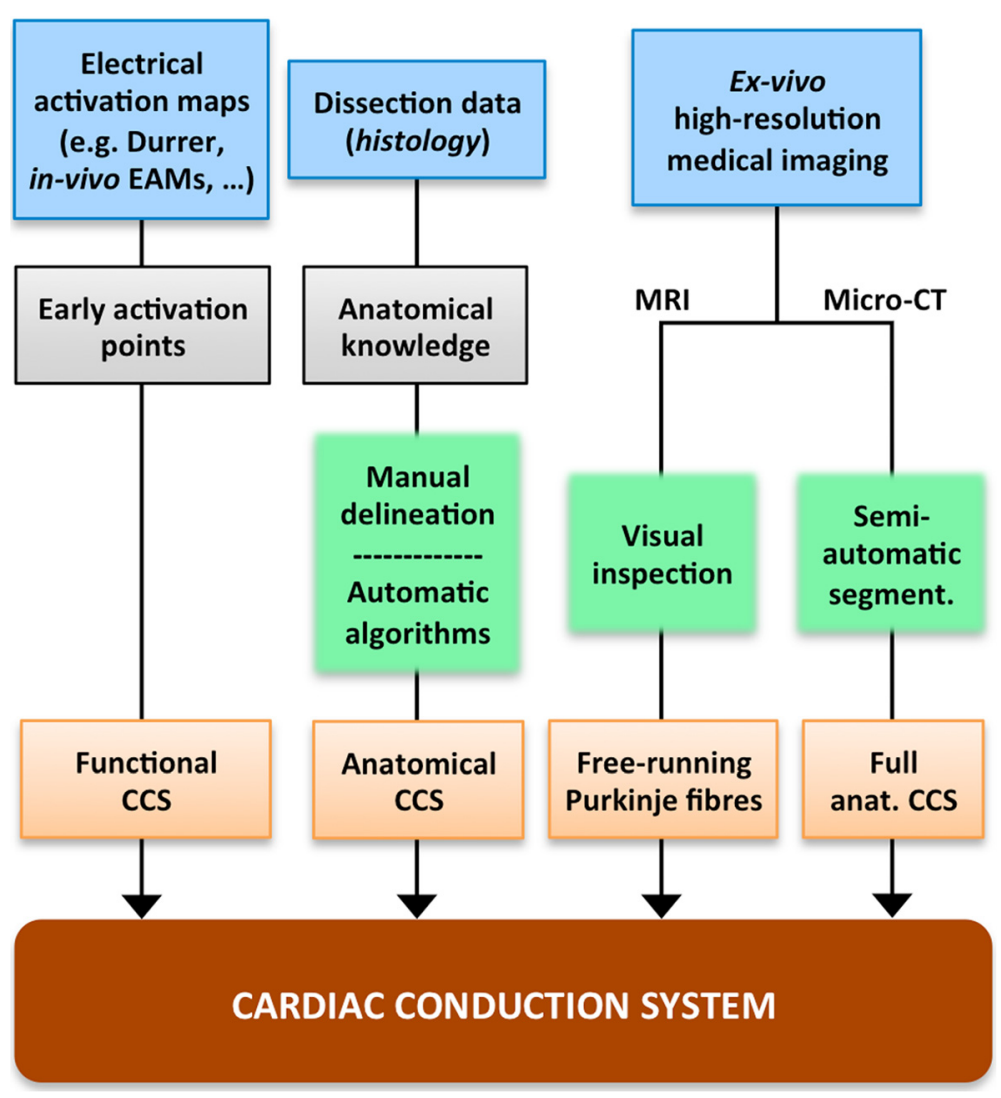

Figure 8 CCS generation stage of the development process of a 3D cardiac computational model. Diagram outlining several ways to generate a model of the CCS to be coupled to a 3D cardiac model. It shows the information sources (blues boxes), the partial results obtained (grey boxes) and the methods (green boxes) used to generate different kinds of model of CCS (orange boxes).

\section{Electrophysiology}

For models aimed at EP computational simulation, once the anatomy and structure of the heart have been defined, a mathematical model that simulates the EP behaviour of the myocardium must be plugged in. Figure 9 briefly summarises the main methods and options to model the cardiac EP using EP models. In the 70s and the 80s, these models usually had the form of cellular automatas [10,101], but these rule-based models were progressively substituted by equation-based ones that consist of two parts: the cellular-level equations and the tissue-level equations (see Figure 9). The cellularlevel equations are based on the well-known Hodgkin and Huxley $(\mathrm{HH})$ formalism established more than 60 years ago [102]. According to this formalism, the cellular AP and the underlying ionic currents are described by a system of non-linear first order ordinary differential equations (ODEs) that models the kinetics of individual ionic channels, pumps and exchangers and the electrical interaction thereof [103]. While these currents are still formulated using the $\mathrm{HH}$ formalism, a new paradigm based on Markov-type models is also being adopted to build more biophysically-based models of ion channels [104]. Over the past decades, extensive patch-clamp experiments that reveal the dynamic properties of ionic channels [105] have provided data to formulate comprehensive mathematical descriptions of ionic currents of different animal species, heart portions and pathophysiological conditions. EP models are now highly specific 


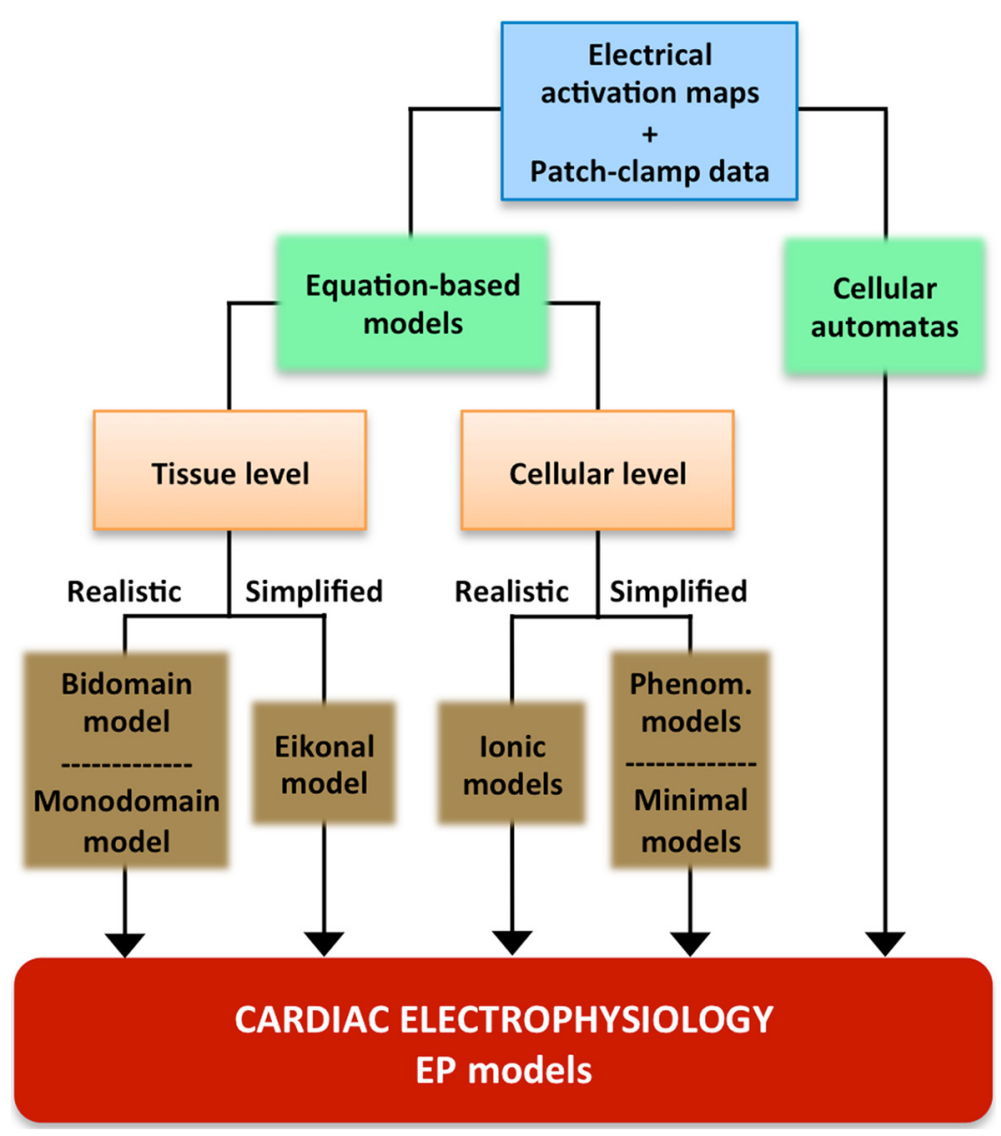

Figure 9 Cardiac electrophysiology modelling stage of the development process of a 3D cardiac computational model. Diagram summarizing very briefly the main methods (green boxes) and options (brown boxes) to model the cardiac EP by means of EP models from EP data (blue box) provided by electrical activation maps at tissue level and patch-clamp experiments at cellular level.

and include human atrial [106], ventricular [107] and Purkinje cells [87] in normal or diseased conditions (see [103] and [108] for review).

The ventricular wall is not homogeneous, as cardiac myocytes in different portions of the ventricles exhibit different ionic currents and APs. Electrophysiological heterogeneities in the ventricles include epicardial-endocardial [109], apico-basal [110] and left-right [111] differences in ion channels and thus in APs. Recent AP models include some of these regional differences; for instance, the last human AP model by O'Hara et al. includes different formulations for endocardial, epicardial and midmyocardial cells [107]. Although there is no experimental evidence on the boundaries of these different regions in the heart anatomy, transmural and other regional differences (e.g. Bachmann's bundle, crista terminalis or pectinate muscles in the atria) in the EP level have been roughly included in some 3D cardiac models [33,112,113].

Myocardial cells are electrically coupled so that current can flow from one cell to neighbouring ones. Thus, the above mentioned cellular models must be accompanied by a tissue-level model in which axial currents flow between cells through lowresistance gap junctions. Such model should take into account the anisotropy inherent to the myocardium, and also the fact that the muscle tissue includes both intracellular 
and extracellular media (domains) separated by cell membranes. A mathematical homogenisation of this concept leads to the so-called bidomain model, which was developed in the late 70s [10]. This model consists of two partial differential equations (PDEs) from which extracellular and intracellular potentials can be derived. Because membrane potentials depend on ionic currents, the tissue-level equations are coupled to the cellular-level ones, so the complete model is formed by a system of two PDEs and a certain number of ODEs, which are strongly non-linear.

The two PDEs include the intracellular and the extracellular conductivity tensors determined by fibre orientation. If equal anisotropy ratios are assumed for the intracellular and extracellular domains (i.e. the two tensors are related by a constant), then the bidomain formulation is reduced to the so-called monodomain approach and the two PDEs become uncoupled [114]. One of the PDEs is of the reaction-diffusion type and includes all the ionic current ODEs in its independent term. Membrane potentials can be obtained by solving this system, while extracellular potentials can be derived directly from the second ODE once membrane potentials are known.

The number of state variables in the ionic AP models (and thus the number of ODEs) can be as high as 48 [107]. The systems of PDEs plus ODEs pose a mathematically challenging problem which must be solved using numerical methods, e.g. FEM method, and which is highly computationally demanding. As a result of these demands, high performance computing techniques are ordinarily used to implement these models. To overcome this limitation, simpler alternatives have been proposed at the cost of losing realism in the mathematical description, such as using the so-called phenomenological models, which reduce the number of state-variables substituting the actual ionic current descriptions by simple mathematical equations. Historically, the first of these simplified models was developed in 1961 by FitzHugh [115], and this approach was further improved and adapted to cardiac AP [116,117]. The mathematical terms of these models have no biophysical correlates in the form of ionic currents, and thus are not feasible to simulate complex dynamical patterns of excitation and repolarisation of cardiac tissue (e.g. rapid pacing and reentrant activity). The so-called minimal models, an evolution of the purely phenomenological models, try to partially overcome this limitation by associating each term to actual but simplified ionic currents $[118,119]$. A different simplification that affects the propagation part of the phenomenon can be adopted by using the so-called Eikonal approximation, which replaces the reaction-diffusion equation with an eikonal equation that is simpler and based on a Huygens approach $[120,121]$. Recently, a new strategy that combines both assumptions (minimal plus Eikonal models) has been proposed [122]. This approach gives rise to a hybrid framework which may combine models with different levels of detail (including detailed biophysical models) whilst maintaining relatively low computational demands [123].

\section{Electromechanical coupling}

Although mentioned, modelling of cardiac mechanics is not addressed in a specific section of this work. Very briefly, it involves the use of biomechanical models at organ level, using the equations of continuum mechanics to describe the deformation of the organ produced by the active tension generated by the myocytes, and models of 
myocyte contraction at cellular level that include myofilaments models at subcellular level depicting the actin-myosin interactions and its calcium-based activation system (e.g. $[124,125])$ (see [126] for a review).

However, it is important to highlight that every $3 \mathrm{D}$ cardiac model aimed at electromechanical (EM) computational simulation must include the EM coupling, also known as excitation-contraction coupling [127,128], as shown in Figure 3. The electrical activation of myocytes is the event that triggers their mechanical contraction by means of the $\mathrm{Ca} 2+$ cycling (the release and reuptake process of intracellular $\mathrm{Ca} 2+$ ), which is the responsible for the initiation of actin-myosin interactions that ultimately lead to myocyte shortening [129]. On the other hand, EM simulation-oriented models can also include the mechanoelectric feedback (see Figure 3). Acute changes in ventricular mechanics can affect cardiac EP [129,130] due to mechanisms such as stretch-activated ion channels [131,132] or mechanical modulation of cell calcium handling, even causing mechanically triggered arrhythmias [133].

\section{Pathology}

There are many diseases that cause structural and/or functional cardiac remodelling, which affects cardiac electrical and/or mechanical performance. Thus, models aimed at studying the effects of those diseases by means of computational simulation should include both types of remodelling. As shown in Figure 3, functional remodelling (electrical or mechanical) may be included in computational simulation-oriented models by adapting the biophysical models that reproduce the behaviour at cell or tissue level. This generally implies altering certain parameters in the equations of the ionic currents to account for the modifications exerted by the remodelling of cardiac tissue [134], or the altered values of certain parameters obtained by patch-clamp experiments at cellular level. A similar approach is used in the case of genetic mutations: their effects on cardiac EP can be mimicked by changing the maximum conductance or the kinetic parameters of the ionic currents directly affected by the mutation [135]. Functional remodelling can also affect the EM coupling. In heart failure, for instance, Ca2+ cycling is altered resulting in impaired contractility $[129,136]$, what increases the risk of extrasystoles and arrhythmia due to the mechanoelectric feedback $[137,138]$. These arrhythmogenic mechanisms may be studied by 3D computational simulations [139].

Structural remodelling can affect cardiac anatomy at organ or tissue level. A pathological variation of cardiac anatomy, which could affect the volume, shape and/or wall thickness of any cardiac chamber, can be taken into account by the geometry of a model, as shown in Figure 3. There are examples of models showing left atrium dilation due to sustained atrial fibrillation [21], right ventricle (RV) hypertrophy [33], hypertrophic and dilated cardiomyopathy in LV [41] or LV wall thinning because of ischaemic injuries [50]. On the other hand, some cardiac pathologies can also alter the myocardial structure and function at tissue level (see Figure 3), which affects the electrical propagation patterns and the mechanical performance. For instance, a 3D cardiac model can include left bundle branch block (LBBB) as a functional alteration at tissue level [140]. Myocardial structure remodelling at tissue level, such as fibre orientation disarray associated to ischaemic 
regions [141], may be also included into a model from ex-vivo DTI [31,53] or histology data [142].

Chronic or healed ischaemic injuries resulting from myocardial infarctions (infarct scars) can be included in order to assess its influence on cardiac performance. These fibrotic lesions are usually segmented from ex-vivo DTI by means of fractional anisotropy [31,53], from anatomical MRI based on wall thinning [50] or more frequently from in-vivo delayed enhancement-MRI (DE-MRI) [143]. Image-based segmentation provides information about the extension and location of an ischaemic injury within the geometry of a given 3D model and also allows differentiating between the core (fibrotic scar) and the border zone (BZ), i.e., the remodelled but still working tissue. However, the associated functional remodelling must be taken into account by the mathematical models used to perform computational simulations, e.g. ionic models for electrical remodelling in the case of EP studies.

Cardiac tissue can also undergo diffuse myocardial fibrosis, which is related to a broad variety of disorders: hypertension, diabetes, dilated and hypertrophic cardiomyopathy, chronic renal insufficiency, atrial fibrillation, etc. The progress of diffuse fibrosis can lead to systolic and diastolic dysfunction [144] and turns the myocardium into a more arrhythmogenic substrate [145]. Therefore, models can include this kind of fibrosis in order to study its influence on the cardiac performance by computational simulation.

\section{Example of a 3D cardiac computational model}

Figure 10 shows an example of a 3D bi-ventricular computational model that includes all the components previously described in this section. The geometry of the ventricles and the scar region are patient-specific, both segmented from the same in-vivo DE-MRI stack. The cardiac geometry was manually segmented with a high level of anatomical detail (see Figure 10a), whilst the infarct scar was semiautomatically delineated by the standard deviation (SD) from remote method [146]. On the contrary, the CCS, the fibre orientation and the biophysical models are generic, i.e., based on population data. The fibre orientation was included by a rulebased algorithm [147] based on the Streeter's findings [72]. An anatomical model of the CCS was generated and coupled to the endocardial surfaces of the bi-ventricular model using an automatic algorithm implemented from dissection data [93]. The FEM volumetric mesh was built using regular hexahedral elements (element size (ds) of $0.4 \mathrm{~mm}$ ) in order to decrease the number of degrees of freedom of the model, yielding a mesh composed by 3.2 million elements and 3.5 million vertices. The final application of this model was computational simulation of cardiac EP using a specific FEM solver called ELVIRA [148], with a time step (dt) of $20 \mu \mathrm{s}$, and different ionic models for myocardium (including transmural heterogeneity) [68] and CCS [87] at cellular level, and monodomian approach [114] at tissue level.

\section{Personalisation of 3D cardiac computational models}

Patient-specific models can open up a new avenue of possibilities in cardiology since they are able to integrate anatomical and functional information of a given patient provided by a variety of techniques (different imaging modalities, invasive electrical 


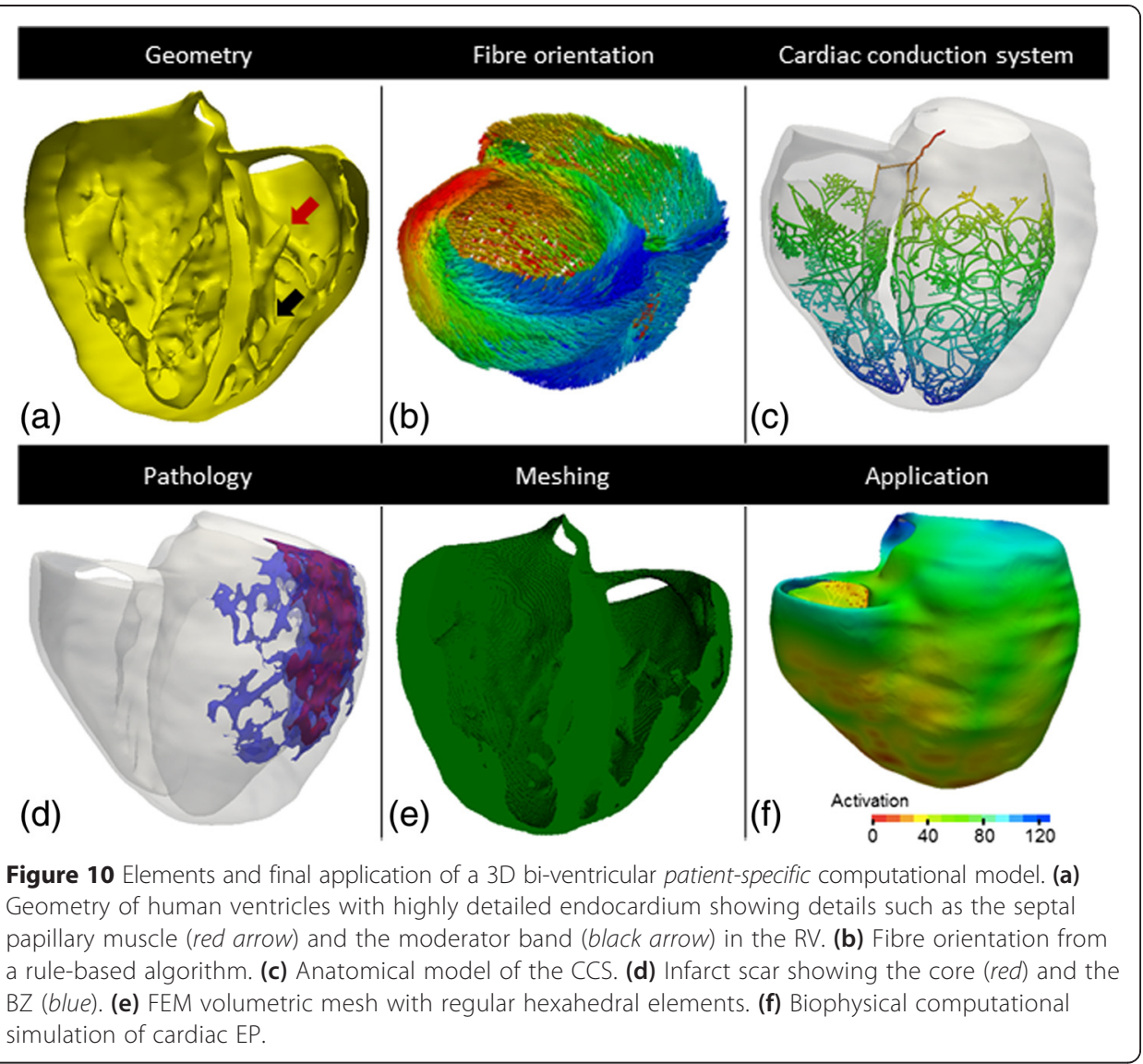

recordings, etc.) in a very comprehensive fashion. This could be very helpful in therapy planning, guidance and follow-up. However, currently only a few cardiac features can be completely personalised. Table 1 summarises the current personalisation possibilities in cardiac computational modelling, which are discussed below.

The anatomy, including pathological anatomical variations, can be personalised for a specific subject by developing a patient-specific model from in-vivo images. Some types of structural remodelling at tissue level can also be personalised, such as the location and extension of infarct scars which can be reconstructed from in-vivo images (e.g. DEMRI) [143] or from EAMs recorded during RFA procedures. T1 mapping is an emerging MRI modality able to quantitatively assess in-vivo the level of diffuse fibrosis $[149,150]$. This novel technique could allow including the patient-specific level of diffuse fibrosis in a 3D cardiac model in a quantitative fashion.

There are two important features that cannot be completely personalised yet: the CCS and the fibre orientation. EAMs can provide the location of some PMJs allowing an inverse estimation of a CCS model that tries to reproduce the patient-specific electrical activation pattern $[99,100]$. Nonetheless, currently there is no in-vivo image modality with enough spatial resolution to enable a detailed visualisation of the CCS structure. For fibre orientation, only sparse 2D DTI slices can be acquired in-vivo to estimate the patient-specific fibre orientation of the LV [78,79].

Regarding cardiac EP, the patient-specific transmembrane current dynamics cannot be measured and hence the mathematical models (e.g. ionic models) that reproduce 
Table 1 Current personalisation possibilities in 3D cardiac computational modelling

\begin{tabular}{|c|c|c|c|}
\hline \multicolumn{2}{|l|}{ Features } & Technique & Invasiveness \\
\hline \multicolumn{2}{|l|}{ Anatomy } & Segmentation of in-vivo image & Non-invasive \\
\hline \multicolumn{2}{|l|}{ Fibre orientation } & Image-based estimation (in-vivo DTI) & Non-invasive \\
\hline \multicolumn{2}{|c|}{ Cardiac conduction system } & PMJs from EAMs & Invasive \\
\hline \multirow{4}{*}{$\begin{array}{l}\text { Pathology } \\
\text { (Structural remodelling) }\end{array}$} & Anatomical variations & Clinical image-based & Non-invasive \\
\hline & \multirow{2}{*}{ Localised fibrosis } & Image-based (e.g. DE-MRI) & Non-invasive \\
\hline & & EAMs & Invasive \\
\hline & Diffuse fibrosis & Image-based (T1 mapping MRI) & Non-invasive \\
\hline \multirow{10}{*}{ Electrophysiology } & Action potential & $\mathrm{NO}$ & \\
\hline & Heterogeneity & NO & \\
\hline & Electrical remodelling & NO & \\
\hline & Genetic mutations & NO & \\
\hline & \multirow{2}{*}{ Conduction velocities } & Global: & Non-invasive \\
\hline & & Local: & Invasive \\
\hline & $A P D$ restitution curve & EAMs & Invasive \\
\hline & Extracellular ion concentrations & $\begin{array}{l}\text { Blood test (electrolyte } \\
\text { concentrations) (time-variant) }\end{array}$ & Invasive \\
\hline & $A$ & ECG or BSPM & Non-invasive \\
\hline & Activation patierm & EAMs & Invasive \\
\hline \multirow{3}{*}{ Cardiac mechanics } & Biomechanical model & Dynamic image-based & Non-invasive \\
\hline & Material properties & NO & \\
\hline & Boundary conditions & Dynamic image-based & Non-invasive \\
\hline
\end{tabular}

This table shows the techniques that can be currently used to personalise the different features and components of a 3D cardiac computational model aimed at biophysical simulation, specifying whether the technique is invasive or non-invasive.

the AP at cellular level cannot be personalised. Due to the same reason, the electrical heterogeneity between different regions (e.g. transmural heterogeneity in ventricular walls), the electrical remodelling or the effects on cardiac EP of a genetic mutation cannot be personalised. However, the EP model at cellular level that best matches the patient's pathology can be chosen from the existing models, obtaining a patient-group instead of a patient-specific personalisation [151,152]. The patient-specific electrical activation patterns and wave propagation conduction velocities $(\mathrm{CV})$ in ventricular myocardium can be estimated for the Eikonal model either globally from ECG or body surface potential maps (BSPM), or locally (as spatially varying parameters) by means of EAMs [153], even taking into account the uncertainty due to the sparsity and noise of clinical data [154]. In [122] an approach to personalise CV and APD restitution curve for bi-ventricular models was proposed by estimating certain EP parameters of a simplified AP model [117] from the patient's ECG and the isochrones provided by in-vivo non-contact endocardial LV mapping [155]. EAMs can also allow locating electrical pathways for a certain subject, such as the main inter-atrial connection $[151,152]$ or the reentrant channels responsible for an infarct-derived ventricular tachycardia (VT) [156,157]. Finally, extracellular ion concentrations can be estimated and set into a model from the measurement of blood electrolyte concentrations, although they are highly time-variant $[151,152]$. 
For cardiac mechanics, the most feasible personalisation approach consists of adjusting some parameters of a biomechanical model using the information obtained by segmenting dynamic images (e.g. cine-MRI, tagged-MRI, dynamic-CT), so that the model is adapted in order to reproduce the patient-specific cardiac motion as accurately as possible [153]. Boundary conditions can be estimated from dynamic images as well.

\section{Applications of 3D cardiac computational models}

Computational clinical cardiology is currently a rising field of research with a large number of potential applications. Cardiac image analysis/segmentation and computational simulation of cardiac physics are two well-established applications of 3D cardiac models. Cardiac EP simulation, for instance, is becoming a powerful tool to gain insight into the electrical cardiac disorders at tissue/organ level. It allows performing in-silico experiments by computational simulation in which all variables are under control and that, in many cases, cannot be carried out in-vivo due to unacceptable risk for the patient, ethical reasons, inability to control all variables, etc. In this section we expose the usefulness of these applications by presenting several examples from the literature mainly related to cardiac EP simulation and model-based segmentation.

\section{Cardiac image segmentation}

One of the most challenging tasks in the development of patient-specific models is the segmentation of in-vivo cardiac images. A wide variety of methods have been developed (see e.g. [158]) but the most advanced approach for the automation of this task is the model-based segmentation. This paradigm has been widely applied to in-vivo cardiac image segmentation and analysis [159]. It requires the use of a reference model so that several kinds of model especially aimed at this purpose have appeared.

Deformable models are based on a template that resembles the target objects, i.e. the cardiac structures to be segmented, which is used as an initialisation of the segmentation process. This template, called initial mesh, can be built using any methodology: ellipsoid-based model [7], image-based model [49,76], assembled from dissection data [160], etc. Briefly, the initial mesh is overlapped to the cardiac image stack to be segmented and then it is deformed driven by the image intensity levels in an iterative process until reaching a given optimum point with the ultimate goal of fitting the initial mesh to the patient-specific geometry.

The mean shape/image resulting from a cardiac atlas can also be used as a segmentation tool by fitting it to the target image, e.g. by non-rigid registration [42]. The so-called statistical cardiac models (or statistical atlases) appeared as an evolution of cardiac atlases. They are a wide range of models mainly represented by statistical shape models $[51,52,54,55]$, although there are other types such as active appearance models $[161,162]$ or active shape models [163]. This model-based segmentation approach relies on an a priori statistical knowledge about cardiac anatomy and/or certain features of cardiac images. This knowledge comes from a statistical characterisation of the anatomical variations (and/or image features) included in the population used to construct a given atlas, usually including both healthy volunteers (normal shaped hearts) and patients with different 
variations of cardiac anatomy. Figure 11 shows the mean shape and the statistical characterisation of a whole-heart statistical atlas [52]. The dimensionality of the resulting variance and co-variance matrix is usually limited using principal components analysis (PCA) $[47,51,54,55]$. The resulting prior knowledge is used in order to enhance the accuracy of the segmentation or analysis process on a specific image modality and to increase its robustness when certain information in the image is poor or missing (see [164] for a review). A mixed approach is the shape-constrained deformable model developed in [47], which includes prior anatomical knowledge provided by a point distribution model (PDM) derived from a cardiac atlas. Although the particular method can vary depending on the type of model, in general the segmentation process is guided by the image information but constrained by the prior knowledge. That is why a statistical model does not consider feasible those anatomical variations that were not learned from the training set. Therefore, the power of a statistical atlas as a segmentation tool strongly depends on the population chosen for the atlas development. Furthermore, most of the statistical models take into account the endocardial surfaces of all cardiac chambers included in the model but only the epicardium of the LV $[47,51,52]$. This is because of the high complexity and variability of the anatomy of the RV and the atria together with the thinness of their walls, what complicates the statistical characterisation of these cardiac chambers. The Cardiac Atlas Project [165], led by the University of Auckland, must be highlighted in this section. It provides a wide database of cardiac images available online which aims to promote a collaborative development of anatomical and functional statistical atlases both for healthy and pathological hearts.

\section{Simulation of acute ischaemia}

In the first 10-15 minutes after coronary artery occlusion (acute ischaemia), changes in ionic currents and concentrations, AP and tissue structure, along with the heterogeneous nature of these changes, predispose the ventricular myocardium to potentially lethal reentrant arrhythmias [166]. In the past decades, the intimate mechanisms of the arrhythmogenicity of acute myocardial ischaemia have been theoretically studied using model-based computational simulation [167]. The effects of acute ischaemia are of

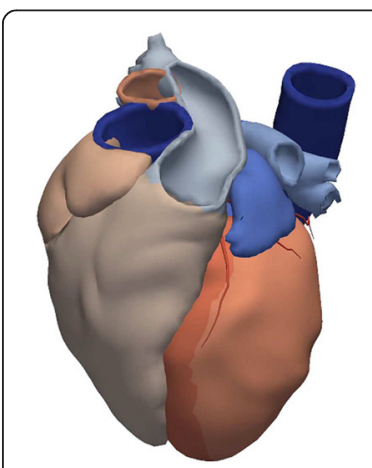

(a)

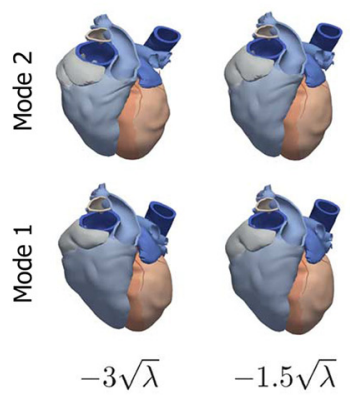

$-3 \sqrt{\lambda}-1.5 \sqrt{\lambda}$

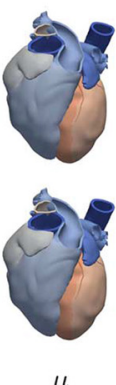

(b)

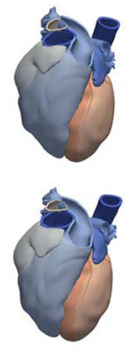

$+1.5 \sqrt{\lambda}$

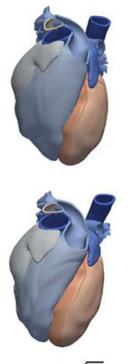

$+3 \sqrt{\lambda}$

Figure 11 Whole-heart cardiac atlas constructed from in-vivo high-resolution MS-CT of 138 human subjects. (a) Mean shape of the whole-heart atlas. (b) First two modes of variation at end diastole of the spatio-temporal whole-heart statistical atlas ( $\mu$ represents the mean shape and $\sqrt{\lambda}$ is the standard deviation). Adapted with permission from [52]. 
multiscale nature [168-173], and the whole-organ effects have recently been analysed using 3D cardiac models.

In [174] a 3D heterogeneous model of regionally ischaemic human ventricles was used to study the dynamics of ischaemic reentrant pathways. The simulation results predicted the appearance of figure-of-eight reentrant wavefronts that cross the central ischaemic zone. These wavefronts are formed in the epicardial surface due to the prolonged refractory period of midmyocardial layers. Also, focal activity experimentally observed in the epicardium could be caused by reentrant wavefronts propagating in the mid-myocardium which re-emerge in the heart surface. Finally, the thin survival layer (wash-out zone) which appears in the endocardial ischaemic BZ protects the myocardium against the perpetuation of reentrant wavefronts that cross the central ischaemic zone.

Global ischaemia has been also simulated in 3D cardiac models in the context of cardiac defibrillation. In [175] a 3D model of rabbit ventricles was used to study the effects of ischaemia on the lower and upper limits of vulnerability to reentry induced by electric shocks. These studies stressed the importance of the transmural electrical events, the spatial extent of the shock-end excitation wavefronts and the slower recovery from shock-induced positive polarisation in the mechanisms responsible for the limits of vulnerability.

\section{Ablation of chronic myocardial infarction}

When the acute phase of ischaemia ends, the ischaemic (now infarcted) tissue heals and ventricular arrhythmias can take place weeks or months after the occlusion [176]. Computer simulations are also of great interest in this chronic period of infarction to aid clinicians during ablation interventions. Examples of simulations of infarcted 3D ventricles are discussed below.

In [177] reentrant mechanisms were simulated on a 3D image-based model of canine infarcted ventricles, including the scar and the BZ with electrical remodelling. The mechanisms of defibrillation efficacy were studied in [178] using a model of rabbit infarcted ventricles. The ability of computer simulations based on DTI images to predict the VT circuits measured in swine EP studies was shown in [179]. Similarly, electrically remodelled BZs and infarct cores were included in [180] in a model of swine heart. VT was induced in different computational models and proved that both infarct scars and peri-infarct zones are needed for VT generation. Similar reentrant circuits were obtained both in computational and EP studies, showing that image-based modelling might be helpful in planning RFA strategies. Indeed, recently the feasibility of 3D simulation based on in-vivo DE-MRI images to estimate ablation targets in human VT has been tested [143,181], emphasizing the effectiveness of this computational tool.

\section{Cardiac resynchronisation therapy}

CRT has become an established therapy to treat certain patients with heart failure suffering from conduction abnormalities such as LBBB. Clinical trials evaluating CRT have demonstrated that $30 \%$ of patients with heart failure and wide QRS do not respond to CRT, especially patients with myocardial infarcts. Computational 
models have been developed to provide insight into the complex mechanism that governs CRT efficacy. In [140] a computer model was designed including different degrees of LBBB (0\%, 20\% and 40\%) for the optimisation of atrioventricular and interventricular (VV) delay, which are key parameters on the CRT device that require a tailored set-up. In $[182,183]$ the effect of the pacing site and infarct location (anterior, inferior, posterolateral, diffuse fibrosis) on regional mechanics and global haemodynamics was studied on an electromechanical dog heart model. They concluded that in hearts with LBBB and large infarcted regions the response to CRT was poorer. The effect of the ventricular morphology (normal, dilated, hypertrophy) on the VV delay in order to obtain an optimal LV synchronisation was studied in [41]. They concluded that the distance between the LV lead of the pacemaker and the CCS (related to thickness of the LV lateral wall) introduces a large delay that needs to be compensated by a pre-activation of the LV lead. In [184] a personalisation strategy for anatomy and function was developed to predict the response to CRT in-silico. Personalised heart models reproduced acute effects of pacing on pressure development for several pacing conditions on two patients, achieving good agreement with invasive haemodynamic measurements. All those computer models were designed with the aim of helping to understand the pathophysiology of asynchrony to further improve CRT.

\section{Conclusions}

3D computational models of cardiac anatomy and function have benefited significantly from the revolution of medical imaging systems. The development of techniques able to build 3D personalised cardiac models is expected to have an impact on diagnosis, therapy planning and prevention of cardiac disorders. The advances on $p a-$ tient-specific modelling have enabled the use of 3D heart models reconstructed from clinical MRI or CT scans. Current 3D cardiac models have a remarkable structural and biophysical detail, and provide a multi-parametric source of information that integrates multimodal images (ex-vivo and in-vivo), one-dimensional signals and biophysical data into a common spatio-temporal coordinate system, which will help to gain insights into several cardiac disorders, e.g. into mechanisms of arrhythmia in many disease settings. Incorporation of critical cardiac structures, such as the CCS, fibre orientation and the coronary tree, will facilitate further biophysical modelling. The next steps towards the translation of this technology into clinical environments are the automation and scalability of model-building procedures, allowing to easily process large-scale image databases, and the evolution of computing technologies such as graphical processing units (GPUs) to speed up the solving process of biophysical simulations.

\section{Additional file}

Additional file 1: Table of reviewed 3D cardiac computational models. Table S1. is a wide summary table that lists the main features included in the sixty 3D cardiac computational models reviewed in this work as well as the material and related methods. It also provides information about the purpose of each model and its online availability. The sixty reviewed models appear in the table sorted by chronological order. Authors strongly encourage the readers to download Additional file 1 in order to get all the information extracted from this review and to be able to compare different models in a straightforward and easy manner. Table S2. shows the meaning of the acronyms and abbreviations used to encode the information contained in Table S1. Additional file 1 also includes some text explaining what kind of information is provided by each column in Table S1 [185-187]. 


\begin{abstract}
Abbreviations
2D: bi-dimensional; 3D: three-dimensional; AP: Action potential; APD: Action potential duration; BSPM: Body surface potential maps; BZ: Border zone; CAD: Computer-aided design; CCS: Cardiac conduction system; CRT: Cardiac resynchronisation therapy; CT: Computed tomography; CV: Conduction velocity; DE-MRl: Delayed enhancement-magnetic resonance imaging; DTI: Diffusion tensor imaging; DT-MRI: Diffusion tensor-magnetic resonance imaging; EAMs: Electroanatomical maps; ECG: Electrocardiogram; EM: Electromechanical; EP: Electrophysiology or electrophysiological; FEM: Finite element method; GPUs: Graphical processing units; HH: Hodgkin and Huxley; LBBB: Left bundle branch block; LV: Left ventricle; MRI: Magnetic resonance imaging; MS-CT: Multi slice-computed tomography; ODEs: Ordinary differential equations; PCA: Principal components analysis; PDEs: Partial differential equations; PDM: Point distribution model; PMJs: Purkinje-myocardium junctions; RFA: Radiofrequency ablation; RV: Right ventricle; SD: Standard deviation; VT: Ventricular tachycardia; W: Interventricular..
\end{abstract}

\title{
Competing interests
}

The authors declare that they have no competing interests.

\section{Authors' contributions}

ALP carried out the bibliographic search and crafted the table from the sixty reviewed 3D cardiac computational models (Additional file 1: Table S1). The three authors have contributed equally in the conception of this work and in the crafting of the manuscript. All authors have read and approved the final manuscript.

\section{Acknowledgements}

This work was partially supported by the "VI Plan Nacional de Investigación Científica, Desarrollo e Innovación Tecnológica" from the Ministerio de Economía y Competitividad of Spain (TIN2012-37546-C03-01 and TIN2011-28067) and the European Commission (European Regional Development Funds - ERDF - FEDER) and by "eTorso project" (GVA/2013-001404) from the Generalitat Valenciana (Spain). ALP is financially supported by the program "Ayudas para contratos predoctorales para la formación de doctores" from the Ministerio de Economía y Competitividad of Spain (BES-2013-064089).

\section{Author details}

${ }^{1}$ Centre for Research and Innovation in Bioengineering (Ci2B), Universitat Politècnica de València, València, Spain.

${ }^{2}$ Computational Multiscale Physiology Lab (CoMMLab), Universitat de València, València, Spain.

Received: 6 October 2014 Accepted: 2 April 2015

\section{Published online: 17 April 2015}

\section{References}

1. Koushanpour E, Collings W. Validation and dynamic applications of an ellipsoid model of the left ventricle. J Appl Physiol. 1966;21:1655-61.

2. Ghista D, Sandler H. An analytic elastic-viscoelastic model for the shape and the forces in the left ventricle. J Biomech. 1969:2:35-47.

3. Janz RF, Grimm AF. Finite-Element Model for the Mechanical Behavior of the Left Ventricle: prediction of deformation in the potassium-arrested rat heart. Circ Res. 1972;30:244-52.

4. Van den Broek JHJM, Van den Broek MHLM. Application of an ellipsoidal heart model in studying left ventricular contractions. J Biomech. 1980:13:493-503.

5. Colli Franzone P, Guerri L, Pennacchio M, Taccardi B. Spread of excitation in 3-D models of the anisotropic cardiac tissue. II. Effects of fiber architecture and ventricular geometry. Math Biosci. 1998;147:131-71.

6. Kerckhoffs RCP, Bovendeerd PHM, Kotte JCS, Prinzen FW, Smits K, Arts T. Homogeneity of cardiac contraction despite physiological asynchrony of depolarization: a model study. Ann Biomed Eng. 2003;31:536-47.

7. Sermesant M, Moireau P, Camara O, Sainte-Marie J, Andriantsimiavona R, Cimrman R, et al. Cardiac function estimation from MRI using a heart model and data assimilation: advances and difficulties. Med Image Anal. 2006;10:642-56

8. Okajima M, Fujino T, Kobayashi T, Yamada K. Computer simulation of the propagation process in excitation of the ventricles. Circ Res. 1968;23:203-11.

9. Horan LG, Hand RC, Johnson JC, Sridharan MR, Rankin TB, Flowers NC. A theoretical examination of ventricular repolarization and the secondary T wave. Circ Res. 1978;42:750-7.

10. Miller WT, Geselowitz DB. Simulation studies of the electrocardiogram. I. The normal heart. Circ Res. 1978;43:301-15.

11. Vetter FJ, McCulloch AD. Three-dimensional analysis of regional cardiac function: a model of rabbit ventricular anatomy. Prog Biophys Mol Biol. 1998;69:157-83.

12. Nielsen PMF, LeGrice IJ, Smaill BH, Hunter PJ. Mathematical model of geometry and fibrous structure of the heart. Am J Physiol Heart Circ Physiol. 1991;260:H1365-78.

13. Stevens C, Remme E, LeGrice I, Hunter P. Ventricular mechanics in diastole: material parameter sensitivity. J Biomech. 2003;36:737-48.

14. Aoki M, Okamoto Y, Musha T, Harumi Kl. Three-dimensional simulation of the ventricular depolarization and repolarization processes and body surface potentials: normal heart and bundle branch block. IEEE Trans Biomed Eng. 1987;34:454-62.

15. Thakor NV, Eisenman LN. Three-dimensional computer model of the heart: fibrillation induced by extrastimulation. Comput Biomed Res. 1989:22:532-45.

16. Freudenberg J, Schiemann T, Tiede U, Höhne KH. Simulation of cardiac excitation patterns in a three-dimensional anatomical heart atlas. Comput Biol Med. 2000;30:191-205.

17. Trunk P, Mocnik J, Trobec R, Gersak B. 3D heart model for computer simulations in cardiac surgery. Comput Biol Med. 2007:37:1398-403

18. Siregar P, Sinteff JP, Julen N, Le Beux P. An interactive 3D anisotropic cellular automata model of the heart. Comput Biomed Res. 1998:31:323-47.

19. Harrild DM, Henriquez CS. A computer model of normal conduction in the human atria. Circ Res. 2000;87:e25-36. 
20. Bodin ON, Kuz'min AV. Synthesis of a realistic model of the surface of the heart. Biomed Eng (NY). 2006;40:280-3.

21. Ruiz-Villa CA, Tobón C, Rodríguez JF, Ferrero JM, Hornero F, Saíz J. Influence of atrial dilatation in the generation of re-entries caused by ectopic activity in the left atrium. Comput Cardiol. 2009;36:457-60.

22. Blanc O, Virag N, Vesin JM, Kappenberger L. A computer model of human atria with reasonable computation load and realistic anatomical properties. IEEE Trans Biomed Eng. 2001;48:1229-37.

23. Zemlin CW, Herzel H, Ho SY, Panfilov AV. A realistic and efficient model of excitation propagation in the human atria. In: Virag N, Kappenberger L, Blanc O, editors. Comput Simul Exp Assess Card Electrophysiol. Arkmonk, New York: Futura Publishing Company, Inc; 2001. p. 29-34.

24. Seemann G, Höper C, Sachse FB, Dössel O, Holden AV, Zhang H. Heterogeneous three-dimensional anatomical and electrophysiological model of human atria. Philos Trans R Soc A Math Phys Eng Sci. 2006;364:1465-81.

25. Zhao J, Butters TD, Zhang H, LeGrice IJ, Sands GB, Smaill BH. Image-based model of atrial anatomy and electrical activation: a computational platform for investigating atrial arrhythmia. IEEE Trans Med Imaging. 2013;32:18-27.

26. Creswell LL, Wyers SG, Pirolo JS, Perman WH, Vannier MW, Pasque MK. Mathematical modeling of the heart using magnetic resonance imaging. IEEE Trans Med Imaging. 1992;11:581-9.

27. Lorange M, Gulrajani RM. A computer heart model incorporating anisotropic propagation: I. Model construction and simulation of normal activation. J Electrocardiol. 1993;26:245-61.

28. Winslow RL, Scollan DF, Holmes A, Yung CK, Zhang J, Jafri MS. Electrophysiological modeling of cardiac ventricular function: from cell to organ. Annu Rev Biomed Eng. 2000;2:119-55.

29. Virag N, Jacquemet V, Henriquez CS, Zozor S, Blanc O, Vesin JM, et al. Study of atrial arrhythmias in a computer model based on magnetic resonance images of human atria. Chaos. 2002;12:754-63.

30. Helm PA, Tseng HJ, Younes L, McVeigh ER, Winslow RL. Ex vivo 3D diffusion tensor imaging and quantification of cardiac laminar structure. Magn Reson Med. 2005;54:850-9.

31. Arevalo HJ, Helm PA, Trayanova NA. Development of a model of the infarcted canine heart that predicts arrhythmia generation from specific cardiac geometry and scar distribution. Comput Cardiol. 2008;35:497-500.

32. Plotkowiak M, Rodriguez B, Plank G, Schneider JE, Gavaghan D, Kohl P, et al. High performance computer simulations of cardiac electrical function based on high resolution MRI datasets. In: Int Conf Comput Sci 2008, LNCS 5101. Berlin Heidelberg: Springer-Verlag; 2008. p. 571-80.

33. Heidenreich EA, Ferrero JM, Doblaré M, Rodríguez JF. Adaptive macro finite elements for the numerical solution of monodomain equations in cardiac electrophysiology. Ann Biomed Eng. 2010;38:2331-45.

34. Gurev V, Lee T, Constantino J, Arevalo H, Trayanova NA. Models of cardiac electromechanics based on individua hearts imaging data: Image-based electromechanical models of the heart. Biomech Model Mechanobiol. 2011:10:295-306

35. Deng D, Jiao P, Ye X, Xia L. An image-based model of the whole human heart with detailed anatomical structure and fiber orientation. Comput Math Methods Med. 2012;2012:16.

36. Aslanidi OV, Nikolaidou T, Zhao J, Smaill BH, Gilbert SH, Holden AV, et al. Application of micro-computed tomography with iodine staining to cardiac imaging, segmentation, and computational model development. IEEE Trans Med Imaging. 2013:32:8-17.

37. Haddad R, Clarysse P, Orkisz M, Croisille P, Revel D, Magnin IE. A realistic anthropomorphic numerical model of the beating heart. In: Funct Imaging Model Heart 2005, LNCS 3504. Berlin Heidelberg: Springer-Verlag; 2005. p. 384-93.

38. Appleton B, Wei Q, Liu N, Xia L, Crozier S, Liu F, et al. An electrical heart model incorporating real geometry and motion. In: 27th Annu Int Conf Eng Med Biol Soc (IEEE-EMBS 2005). Shanghai, China: IEEE; 2006. p. 345-8.

39. Niederer S, Rhode K, Razavi R, Smith N. The importance of model parameters and boundary conditions in whole organ models of cardiac contraction. In: Funct Imaging Model Heart 2009, LNCS 5528. Berlin Heidelberg: Springer-Verlag; 2009. p. 348-56.

40. Yang G, Toumoulin C, Coatrieux JL, Shu H, Luo L, Boulmier D. A 3D static heart model from a MSCT data set. In: 27th Annu Int Conf IEEE Eng Med Biol Soc (IEEE-EMBS 2005). Shangai, China: IEEE; 2006. p. 5499-502.

41. Romero D, Sebastian R, Bijnens BH, Zimmerman V, Boyle PM, Vigmond EJ, et al. Effects of the purkinje system and cardiac geometry on biventricular pacing: a model study. Ann Biomed Eng. 2010;38:1388-98.

42. Lorenzo-Valdés M, Sanchez-Ortiz Gl, Mohiaddin R, Rueckert D. Atlas-based segmentation and tracking of 3D cardiac MR images using non-rigid registration. In: Med Image Comput Comput Assist Interv 2002, LNCS 2488. Berlin Heidelberg: Springer-Verlag; 2002. p. 642-50.

43. Ordas S, Oubel E, Sebastian R, Frangi AF. Computational anatomy atlas of the heart. In: 5th Int Symp Image Signal Process Anal (ISPA 2007). Istanbul, Turkey: IEEE; 2007. p. 338-42.

44. Burton RAB, Plank G, Schneider JE, Grau V, Ahammer H, Keeling SL, et al. Three-dimensional models of individual cardiac histoanatomy: tools and challenges. Ann N Y Acad Sci. 2006;1080:301-19.

45. Plank G, Burton RAB, Hales P, Bishop M, Mansoori T, Bernabeu MO, et al. Generation of histo-anatomically representative models of the individual heart: tools and application. Philos Trans R Soc A Math Phys Eng Sci. 2009;367:2257-92.

46. Bishop MJ, Plank G, Burton RAB, Schneider JE, Gavaghan DJ, Grau V, et al. Development of an anatomically detailed MRI-derived rabbit ventricular model and assessment of its impact on simulations of electrophysiological function. Am J Physiol - Heart Circ Physiol. 2010;298:H699-718.

47. Ecabert O, Peters J, Schramm H, Lorenz C, von Berg J, Walker MJ, et al. Automatic model-based segmentation of the heart in CT images. IEEE Trans Med Imaging. 2008;27:1189-201.

48. Ecabert O, Peters J, Walker MJ, Ivanc T, Lorenz C, von Berg J, et al. Segmentation of the heart and great vessels in CT images using a model-based adaptation framework. Med Image Anal. 2011;15:863-76.

49. Schulte RF, Sands GB, Sachse FB, Dössel O, Pullan AJ. Creation of a human heart model and its customisation using ultrasound images. Biomed Tech Eng. 2001;46:26-8.

50. Wenk JF, Zhang Z, Cheng G, Malhotra D, Acevedo-Bolton G, Burger M, et al. First finite element model of the left ventricle with mitral valve: insights into ischemic mitral regurgitation. Ann Thorac Surg. 2010;89:1546-53.

51. Frangi AF, Rueckert D, Schnabel JA, Niessen WJ. Automatic construction of multiple-object three-dimensional statistical shape models: application to cardiac modeling. IEEE Trans Med Imaging. 2002;21:1151-66. 
52. Hoogendoorn C, Duchateau N, Sánchez-Quintana D, Whitmarsh T, Sukno FM, De Craene M, et al. A high-resolution atlas and statistical model of the human heart from multislice CT. IEEE Trans Med Imaging. 2013;32:28-44.

53. Vadakkumpadan F, Rantner LJ, Tice B, Boyle P, Prassl AJ, Vigmond E, et al. Image-based models of cardiac structure with applications in arrhythmia and defibrillation studies. J Electrocardiol. 2009;42:157. e1.

54. Perperidis D, Mohiaddin R, Rueckert D. Construction of a $4 \mathrm{D}$ statistical atlas of the cardiac anatomy and its use in classification. In: Med Image Comput Comput Interv 2005, LNCS 3750. Berlin Heidelberg: Springer-Verlag; 2005. p. 402-10.

55. Lötjönen J, Kivistö S, Koikkalainen J, Smutek D, Lauerma K. Statistical shape model of atria, ventricles and epicardium from short- and long-axis MR images. Med Image Anal. 2004;8:371-86.

56. Lorenz C, von Berg J. A comprehensive shape model of the heart. Med Image Anal. 2006;10:657-70.

57. Mansoori T, Plank G, Burton R, Schneider J, Khol P, Gavaghan D, et al. An iterative method for registration of high-resolution cardiac histoanatomical and MRI images. In: 4th IEEE Int Symp Biomed Imaging: From Nano to Macro (ISBI 2007). Arlington, VA (USA): IEEE; 2007. p. 572-5.

58. Gibb M, Burton RAB, Bollensdorff C, Afonso C, Mansoori T, Schotten U, et al. Resolving the three-dimensional histology of the heart. In: Comput Methods Syst Biol - Lect Notes Comput Sci 7605. Berlin Heidelberg: Springer; 2012. p. 2-16

59. Burton RAB, Lee P, Casero R, Garny A, Siedlecka U, Schneider JE, et al. Three-dimensional histology: tools and application to quantitative assessment of cell-type distribution in rabbit heart. Europace. 2014;16 Suppl 4:iv86-95.

60. Niederer SA, Shetty AK, Plank G, Bostock J, Razavi R, Smith NP, et al. Biophysical modeling to simulate the response to multisite left ventricular stimulation using a quadripolar pacing lead. Pacing Clin Electrophysiol. 2012;35:204-14.

61. Weese J, Groth A, Nickisch H, Barschdorf H, Weber FM, Velut J, et al. Generating anatomical models of the heart and the aorta from medical images for personalized physiological simulations. Med Biol Eng Comput. 2013:51:1209-19.

62. Gibb M, Bishop M, Burton R, Kohl P, Grau V, Plank G, et al. The role of blood vessels in rabbit propagation dynamics and cardiac arrhythmias. In: Funct Imaging Model Heart - FIMH 2009, LNCS 5528. Berlin Heidelberg: Springer; 2009. p. 268-76.

63. Prassl AJ, Kickinger F, Ahammer H, Grau V, Schneider JE, Hofer E, et al. Automatically generated, anatomically accurate meshes for cardiac electrophysiology problems. IEEE Trans Biomed Eng. 2009;56:1318-30.

64. Dux-Santoy L, Sebastian R, Felix-Rodriguez J, Ferrero JM, Saiz J. Interaction of specialized cardiac conduction system with antiarrhythmic drugs: a simulation study. IEEE Trans Biomed Eng. 2011;58:3475-8.

65. Lamata P, Niederer S, Nordsletten D, Barber DC, Roy I, Hose DR, et al. An accurate, fast and robust method to generate patient-specific cubic Hermite meshes. Med Image Anal. 2011;15:801-13.

66. Pathmanathan P, Cooper J, Fletcher A, Mirams G, Murray P, Osborne J, et al. A computational study of discrete mechanical tissue models. Phys Biol. 2009;6:036001.

67. Niederer SA, Kerfoot E, Benson AP, Bernabeu MO, Bernus O, Bradley C, et al. Verification of cardiac tissue electrophysiology simulators using an N-version benchmark. Philos Trans R Soc A Math Phys Eng Sci. 2011;369:4331-51.

68. Ten Tusscher KHWJ, Panfilov AV. Cell model for efficient simulation of wave propagation in human ventricular tissue under normal and pathological conditions. Phys Med Biol. 2006;51:6141-56.

69. LeGrice I, Smaill B, Chai L, Edgar S, Gavin J, Hunter P. Laminar structure of the heart: ventricular myocyte arrangement and connective tissue architecture in the dog. Am J Physiol Heart Circ Physiol. 1995;269:H571-82.

70. Anderson RH, Smerup M, Sanchez-Quintana D, Loukas M, Lunkenheimer PP. The three-dimensional arrangement of the myocytes in the ventricular walls. Clin Anat. 2009;22:64-76.

71. Clerc L. Directional differences of impulse spread in trabecular muscle from mammalian heart. J Physiol. 1976;255:335-46.

72. Streeter Jr DD, Spotnitz HM, Patel DP, Ross Jr J, Sonnenblick EH. Fiber orientation in the canine left ventricle during diastole and systole. Circ Res. 1969;24:339-47.

73. Scollan D, Holmes A, Winslow R, Forder J. Histological validation of myocardial microstructure obtained from diffusion tensor magnetic resonance imaging. Am J Physiol Heart Circ Physiol. 1998;275:H2308-18.

74. Hsu EW, Muzikant AL, Matulevicius SA, Penland RC, Henriquez CS. Magnetic resonance myocardial fiber-orientation mapping with direct histological correlation. Am J Physiol Heart Circ Physiol. 1998;274:H1627-34.

75. Holmes AA, Scollan DF, Winslow RL. Direct histological validation of diffusion tensor MRI in formaldehyde-fixed myocardium. Magn Reson Med. 2000;44:157-61.

76. Sermesant M, Forest C, Pennec X, Delingette H, Ayache N. Deformable biomechanical models: application to 4D cardiac image analysis. Med Image Anal. 2003;7:475-88.

77. Peyrat JM, Sermesant M, Pennec X, Delingette H, Xu C, McVeigh ER, et al. A computational framework for the statistical analysis of cardiac diffusion tensors: application to a small database of canine hearts. IEEE Trans Med Imaging. 2007;26:1500-14.

78. Toussaint N, Sermesant M, Stoeck CT, Kozerke S, Batchelor PG. In vivo human 3D cardiac fibre architecture: reconstruction using curvilinear interpolation of diffusion tensor images. Med Image Comput Comput Assist Interv. 2010;13(Pt 1):418-25.

79. Toussaint N, Stoeck CT, Schaeffter T, Kozerke S, Sermesant M, Batchelor PG. In vivo human cardiac fibre architecture estimation using shape-based diffusion tensor processing. Med Image Anal. 2013;17:1243-55.

80. Bishop MJ, Hales P, Plank G, Gavaghan DJ, Scheider J, Grau V. Comparison of rule-based and DTMRI-derived fibre architecture in a whole rat ventricular computational model. In: Funct Imaging Model Heart 2009, LNCS 5528. Berlin Heidelberg: Springer-Verlag; 2009. p. 87-96.

81. Bayer JD, Blake RC, Plank G, Trayanova NA. A novel rule-based algorithm for assigning myocardial fiber orientation to computational heart models. Ann Biomed Eng. 2012;40:2243-54.

82. Dobrzynski H, Anderson RH, Atkinson A, Borbas Z, D'Souza A, Fraser JF, et al. Structure, function and clinical relevance of the cardiac conduction system, including the atrioventricular ring and outflow tract tissues. Pharmacol Ther. 2013;139:260-88. 
83. Tranum-Jensen J, Wilde AA, Vermeulen JT, Janse MJ. Morphology of electrophysiologically identified junctions between Purkinje fibers and ventricular muscle in rabbit and pig hearts. Circ Res. 1991;69:429-37.

84. Boyle PM, Deo M, Plank G, Vigmond EJ. Purkinje-mediated effects in the response of quiescent ventricles to defibrillation shocks. Ann Biomed Eng. 2010;38:456-68.

85. Behradfar E, Nygren A, Vigmond EJ. The role of Purkinje-myocardial coupling during ventricular arrhythmia: a modeling study. PLoS One. 2014;9, e88000.

86. Difrancesco D, Noble D. A model of cardiac electrical activity incorporating ionic pumps and concentration changes. Philos Trans R Soc B Biol Sci. 1985;307:353-98.

87. Stewart P, Aslanidi OV, Noble D, Noble PJ, Boyett MR, Zhang H. Mathematical models of the electrical action potential of Purkinje fibre cells. Philos Trans R Soc A Math Phys Eng Sci. 2009;367:2225-55.

88. Li P, Rudy Y. A model of canine purkinje cell electrophysiology and $\mathrm{Ca}(2+)$ cycling: rate dependence, triggered activity, and comparison to ventricular myocytes. Circ Res. 2011;109:71-9.

89. Chinchapatnam P, Rhode KS, Ginks M, Mansi T, Peyrat JM, Lambiase P, et al. Estimation of volumetric myocardial apparent conductivity from endocardial electro-anatomical mapping. In: 31st Annu Int Conf IEEE Eng Med Biol Soc (EMBC 2009). Minneapolis, MN (USA): IEEE; 2009. p. 2907-10.

90. Durrer D, Van Dam RT, Freud GE, Janse MJ, Meijler FL, Arzbaecher RC. Total excitation of the isolated human heart. Circulation. 1970;41:899-912.

91. Pollard AE, Barr RC. Computer simulations of activation in an anatomically based model of the human ventricular conduction system. IEEE Trans Biomed Eng. 1991;38:982-96.

92. Abboud S, Berenfeld O, Sadeh D. Simulation of high-resolution QRS complex using a ventricular model with a fractal conduction system. Effects of ischemia on high-frequency QRS potentials. Circ Res. 1991;68:1751-60.

93. Sebastian R, Zimmerman V, Romero D, Sanchez-Quintana D, Frangi AF. Characterization and modeling of the peripheral cardiac conduction system. IEEE Trans Med Imaging. 2013;32:45-55.

94. Bordas R, Gillow K, Lou Q, Efimov IR, Gavaghan D, Kohl P, et al. Rabbit-specific ventricular model of cardiac electrophysiological function including specialized conduction system. Prog Biophys Mol Biol. 2011;107:90-100.

95. Stephenson RS, Boyett MR, Hart G, Nikolaidou T, Cai X, Corno AF, et al. Contrast enhanced micro-computed tomography resolves the 3-dimensional morphology of the cardiac conduction system in mammalian hearts. PLoS One. 2012;7, e35299.

96. Berenfeld $\mathrm{O}$, Jalife J. Purkinje-Muscle reentry as a mechanism of polymorphic ventricular arrhythmias in a 3-dimensional model of the ventricles. Circ Res. 1998;82:1063-77.

97. Azzouzi A, Coudière Y, Turpault R, Zemzemi N. A mathematical model of the Purkinje-muscle junctions. Math Biosci Eng MBE. 2011:8:915-30.

98. Dux-Santoy L, Sebastian R, Rodriguez JF, Ferrero JM. Modeling the different sections of the cardiac conduction system to obtain realistic electrocardiograms. In: 35th Annu Int Conf IEEE Eng Med Biol Soc (EMBC 2013). Osaka, Japan: IEEE; 2013. p. 6846-9.

99. Cardenes R, Sebastian R, Berruezo A, Camara O. Inverse estimation of ventricular Purkinje tree pathways from sequences of depolarization. In: Comput Cardiol. Volume 41. Cambridge, Massachusetts (USA): IEEE; 2014. p. 677-80.

100. Palamara S, Vergara C, Catanzariti D, Faggiano E, Pangrazzi C, Centonze M, et al. Computational generation of the Purkinje network driven by clinical measurements: the case of pathological propagations. Int J Numer Method Biomed Eng. 2014;30:1558-77.

101. Moe GK, Rheinboldt WC, Abildskov JA. A computer model of atrial fibrillation. Am Heart J. 1964;67:200-20.

102. Hodgkin AL, Huxley AF. A quantitative description of membrane current and its application to conduction and excitation in nerve. J Physiol. 1952;117:500-44.

103. Fink M, Niederer SA, Cherry EM, Fenton FH, Koivumäki JT, Seemann G, et al. Cardiac cell modelling: Observations from the heart of the cardiac physiome project. Prog Biophys Mol Biol. 2011;104:2-21.

104. Rudy Y, Silva JR. Computational biology in the study of cardiac ion channels and cell electrophysiology. Q Rev Biophys. 2006:39:57-116.

105. Sakmann B, Neher E. Patch clamp techniques for studying ionic channels in excitable membranes. Annu Rev Physiol. 1984;41:455-72.

106. Maleckar MM, Greenstein JL, Giles WR, Trayanova NA. K+ current changes account for the rate dependence of the action potential in the human atrial myocyte. Am J Physiol Heart Circ Physiol. 2009;297:H1398-410.

107. O'Hara T, Virág L, Varró A, Rudy Y. Simulation of the undiseased human cardiac ventricular action potential: model formulation and experimental validation. PLoS Comput Biol. 2011;7, e1002061.

108. Roberts BN, Yang PC, Behrens SB, Moreno JD, Clancy CE. Computational approaches to understand cardiac electrophysiology and arrhythmias. Am J Physiol Heart Circ Physiol. 2012;303:H766-83.

109. Liu DW, Gintant GA, Antzelevitch C. Ionic bases for electrophysiological distinctions among epicardial, midmyocardial, and endocardial myocytes from the free wall of the canine left ventricle. Circ Res. 1993;72:671-87.

110. Szentadrassy N, Banyasz T, Biro T, Szabo G, Toth Bl, Magyar J, et al. Apico-basal inhomogeneity in distribution of ion channels in canine and human ventricular myocardium. Cardiovasc Res. 2005;65:851-60.

111. Volders PG, Sipido KR, Carmeliet E, Spätjens RL, Wellens HJ, Vos MA. Repolarizing K+ currents ITO1 and IKs are larger in right than left canine ventricular midmyocardium. Circulation. 1999;99:206-10.

112. Tobón C, Rodríguez JF, Ferrero Jr JM, Hornero F, Saiz J. Dominant frequency and organization index maps in a realistic three-dimensional computational model of atrial fibrillation. Europace. 2012;14:v25-32.

113. Tobón C, Ruiz-Villa CA, Heidenreich E, Romero L, Hornero F, Saiz J. A three-dimensional human atrial model with fiber orientation. Electrograms and arrhythmic activation patterns relationship. PLoS One. 2013;8, e50883.

114. Roth BJ. The electrical potential produced by a strand of cardiac muscle: a bidomain analysis. Ann Biomed Eng. 1988;16:609-37.

115. FitzHugh R. Impulses and physiological states in theoretical models of nerve membrane. Biophys J. 1961;1:445-66.

116. Aliev RR, Panfilov AV. Modeling of heart excitation patterns caused by a local inhomogeneity. J Theor Biol. 1996;181:33-40.

117. Mitchell CC, Schaeffer DG. A two-current model for the dynamics of cardiac membrane. Bull Math Biol 2003:65:767-93. 
118. Fenton F, Karma A. Vortex dynamics in three-dimensional continuous myocardium with fiber rotation: filament instability and fibrillation. Chaos. 1998:8:20-47.

119. Bueno-Orovio A, Cherry EM, Fenton FH. Minimal model for human ventricular action potentials in tissue. J Theor Biol. 2008:253:544-60

120. Colli Franzone P, Guerri L, Rovida S. Wavefront propagation in an activation model of the anisotropic cardiac tissue: asymptotic analysis and numerical simulations. J Math Biol. 1990;28:121-76.

121. Keener JP. An eikonal-curvature equation for action potential propagation in myocardium. J Math Biol. 1991;29:629-51.

122. Relan J, Chinchapatnam P, Sermesant M, Rhode K, Ginks M, Delingette H, et al. Coupled personalization of cardiac electrophysiology models for prediction of ischaemic ventricular tachycardia. Interface Focus. 2011;1:396-407.

123. Camara O, Sermesant M, Lamata P, Wang L, Pop M, Relan J, et al. Inter-model consistency and complementarity: Learning from ex-vivo imaging and electrophysiological data towards an integrated understanding of cardiac physiology. Prog Biophys Mol Biol. 2011;107:122-33.

124. Rice JJ, Wang F, Bers DM, de Tombe PP. Approximate model of cooperative activation and crossbridge cycling in cardiac muscle using ordinary differential equations. Biophys J. 2008;95:2368-90.

125. Mullins PD, Bondarenko VE. A mathematical model of the mouse ventricular myocyte contraction. PLoS One. 2013:8:e63141.

126. Trayanova NA, Rice JJ. Cardiac electromechanical models: from cell to organ. Front Physiol. 2011;2:Article 43

127. Williams GSB, Smith GD, Sobie EA, Jafri MS. Models of cardiac excitation-contraction coupling in ventricular myocytes. Math Biosci. 2010:226:1-15.

128. Jafri MS. Models of excitation-contraction coupling in cardiac ventricular myocytes. In: Bioinforma Drug Discov Methods Mol Biol, vol. 910. New York: Springer Science + Business Media; 2012. p. 309-35.

129. Pfeiffer ER, Tangney JR, Omens JH, McCulloch AD. Biomechanics of cardiac electromechanical coupling and mechanoelectric feedback. J Biomech Eng. 2014;136:021007-1-021007-11.

130. Quinn TA, Kohl P, Ravens U. Cardiac mechano-electric coupling research: fifty years of progress and scientific innovation. Prog Biophys Mol Biol. 2014;115:71-5.

131. Craelius W, Chen V, El-Sherif N. Stretch activated ion channels in ventricular myocytes. Biosci Rep. 1988;8:407-14.

132. Kohl P, Hunter P, Noble D. Stretch-induced changes in heart rate and rhythm: clinical observations, experiments and mathematical models. Prog Biophys Mol Biol. 1999;71:91-138

133. Kohl P, Sachs F, Franz MR. Cardiac Mechano-Electric Feedback and Arrhytmias: From Pipette to Patient. Philadelphia: Elsevier Health Sciences; 2005.

134. Cabo C, Boyden PA. Electrical remodeling of the epicardial border zone in the canine infarcted heart: a computational analysis. Am J Physiol Heart Circ Physiol. 2003;284:H372-84.

135. Clancy CE, Rudy Y. Cellular consequences of HERG mutations in the long QT syndrome: precursors to sudden cardiac death. Cardiovasc Res. 2001;50:301-13.

136. Marks AR. Calcium cycling proteins and heart failure: mechanisms and therapeutics. J Clin Invest. 2013;123:46-52.

137. Hansen DE, Craig CS, Hondeghem LM. Stretch-induced arrhythmias in the isolated canine ventricle. Evidence for the importance of mechanoelectrical feedback. Circulation. 1990;81:1094-105.

138. Wang Z, Taylor LK, Denney WD, Hansen DE. Initiation of ventricular extrasystoles by myocardial stretch in chronically dilated and failing canine left ventricle. Circulation. 1994;90:2022-31.

139. Trayanova NA, Constantino J, Gurev V. Models of stretch-activated ventricular arrhythmias. J Electrocardiol. 2010;43:476-85.

140. Reumann M, Farina D, Miri R, Lurz S, Osswald B, Dössel O. Computer model for the optimization of AV and W delay in cardiac resynchronization therapy. Med Biol Eng Comput. 2007:45:845-54.

141. Wu M-T, Tseng W-YI SM-YM, Liu C-P, Chiou K-R, Wedeen VJ, Reese TG, et al. Diffusion tensor magnetic resonance imaging mapping the fiber architecture remodeling in human myocardium after infarction: correlation with viability and wall motion. Circulation. 2006;114:1036-45.

142. Rutherford SL, Trew ML, Sands GB, LeGrice IJ, Smaill BH. High-resolution 3-dimensional reconstruction of the infarct border zone: impact of structural remodeling on electrical activation. Circ Res. 2012;111:301-11.

143. Ashikaga H, Arevalo H, Vadakkumpadan F, Blake RC, Bayer JD, Nazarian S, et al. Feasibility of image-based simulation to estimate ablation target in human ventricular arrhythmia. Heart Rhythm. 2013;10:1109-16.

144. Martos R, Baugh J, Ledwidge M, O'Loughlin C, Conlon C, Patle A, et al. Diastolic heart failure: evidence of increased myocardial collagen turnover linked to diastolic dysfunction. Circulation. 2007;115:888-95.

145. Ten Tusscher KHWJ, Panfilov AV. Influence of diffuse fibrosis on wave propagation in human ventricular tissue. Europace. 2007;9 Suppl 6:vi38-45

146. Kim RJ, Fieno DS, Parrish TB, Harris K, Chen EL, Simonetti O, et al. Relationship of MRI delayed contrast enhancement to irreversible injury, infarct age, and contractile function. Circulation. 1999;100:1992-2002.

147. Sebastian R, Zimmerman V, Sukno F, Bijnens BH, Frangi AF. Cardiac modelling for pathophysiology research and clinical applications. The need for an automated pipeline. In: World Congr Med Phys Biomed Eng 2009. Berlin Heidelberg: Springer; 2010. p. 2207-10.

148. Heidenreich EA, Gaspar FJ, Ferrero JM, Rodríquez JF. Compact schemes for anisotropic reaction-diffusion equations with adaptive time step. Int J Numer Methods Eng. 2010;82:1022-43.

149. Mewton N, Liu CY, Croisille P, Bluemke D, Lima JAC. Assessment of myocardial fibrosis with cardiovascular magnetic resonance. J Am Coll Cardiol. 2011;57:891-903.

150. Dass S, Suttie JJ, Piechnik SK, Ferreira VM, Holloway CJ, Banerjee R, et al. Myocardial tissue characterization using magnetic resonance noncontrast T1 mapping in hypertrophic and dilated cardiomyopathy. Circ Cardiovasc Imaging. 2012;5:726-33.

151. Krueger MW, Seemann G, Rhode K, Keller DUJ, Schilling C, Arujuna A, et al. Personalization of atrial anatomy and electrophysiology as a basis for clinical modeling of radio-frequency ablation of atrial fibrillation. IEEE Trans Med Imaging. 2013;32:73-84

152. Krueger MW, Schulze WHW, Rhode KS, Razavi R, Seemann G, Dössel O. Towards personalized clinical in-silico modeling of atrial anatomy and electrophysiology. Med Biol Eng Comput. 2013;51:1251-60. 
153. Sermesant M, Peyrat J-M, Chinchapatnam P, Billet F, Mansi T, Rhode K, et al. Toward patient-specific myocardial models of the heart. Heart Fail Clin. 2008;4:289-301.

154. Konukoglu E, Relan J, Cilingir U, Menze BH, Chinchapatnam P, Jadidi A, et al. Efficient probabilistic model personalization integrating uncertainty on data and parameters: Application to eikonal-diffusion models in cardiac electrophysiology. Prog Biophys Mol Biol. 2011;107:134-46.

155. Lambiase PD, Rinaldi A, Hauck J, Mobb M, Gill JS, Bucknall CA. Non-contact left ventricular endocardial mapping in cardiac resynchronisation therapy. Heart. 2004;90:44-51.

156. Perez-David E, Arenal A, Rubio-Guivernau JL, del Castillo R, Atea L, Arbelo E, et al. Noninvasive identification of ventricular tachycardia-related conducting channels using contrast-enhanced magnetic resonance imaging in patients with chronic myocardial infarction: comparison of signal intensity scar mapping and endocardial voltage mappin. J Am Coll Cardiol. 2011:57:184-94.

157. Fernández-Armenta J, Berruezo A, Andreu D, Camara O, Silva E, Serra L, et al. Three-dimensional architecture of scar and conducting channels based on high resolution ce-CMR: insights for ventricular tachycardia ablation. Circ Arrhythm Electrophysiol. 2013;6:528-37.

158. Petitjean C, Dacher JN. A review of segmentation methods in short axis cardiac MR images. Med Image Anal. 2011;15:169-84.

159. Frangi AF, Niessen WJ, Viergever MA. Three-dimensional modeling for functional analysis of cardiac images: a review. IEEE Trans Med Imaging. 2001;20:2-25.

160. Sermesant M, Delingette $H$, Ayache N. An electromechanical model of the heart for image analysis and simulation. IEEE Trans Med Imaging. 2006;25:612-25.

161. Mitchell S, Lelieveldt B, van der Geest R, Bosch J, Reiber J, Sonka M. Segmentation of cardiac MR images: An active appearance model approach. In: Hanson KM, editor. Proc SPIE 3979, Med Imaging 2000: Image Process. San Diego, CA (USA): SPIE Digital Library; 2000. p. 224-34.

162. Mitchell SC, Bosch JG, Lelieveldt BPF, van der Geest RJ, Reiber JHC, Sonka M. 3-D active appearance models: segmentation of cardiac MR and ultrasound images. IEEE Trans Med Imaging. 2002;21:1167-78.

163. Ordas S, Boisrobert L, Huguet M, Frangi AF. Active shape models with invariant optimal features (IOF-ASM) application to cardiac MRI segmentation. In: Comput Cardiol. Volume 30. Thessaloniki, Greece: IEEE; 2003. p. 633-6.

164. Young AA, Frangi AF. Computational cardiac atlases: from patient to population and back. Exp Physiol. 2009:94:578-96.

165. Fonseca CG, Backhaus M, Bluemke DA, Britten RD, Chung JDO, Cowan BR, et al. The Cardiac Atlas Project - an imaging database for computational modeling and statistical atlases of the heart. Bioinformatics. 2011;27:2288-95.

166. Janse MJ, Kleber AG. Electrophysiological changes and ventricular arrhythmias in the early phase of regional myocardial ischemia. Circ Res. 1981;49:1069-81.

167. Ferrero JM, Trenor B, Romero L. Multiscale computational analysis of the bioelectric consequences of myocardial ischaemia and infarction. Europace. 2014;16:405-15.

168. Ferrero Jr JM, Sáiz J, Ferrero JM, Thakor NV. Simulation of action potentials from metabolically impaired cardiac myocytes: role of ATP-sensitive K+ current. Circ Res. 1996;79:208-21.

169. Ferrero Jr JM, Trénor B, Rodríguez B, Sáiz J. Electrical activity and reentry during acute regional myocardial ischemia: insights from simulations. Int J Bifurc Chaos. 2003;13:3703-15.

170. Shaw RM, Rudy Y. Electrophysiologic effects of acute myocardial ischemia: a theoretical study of altered cell excitability and action potential duration. Cardiovasc Res. 1997;35:256-72.

171. Trénor B, Romero L, Ferrero JM, Sáiz J, Moltó G, Alonso JM. Vulnerability to reentry in a regionally ischemic tissue: a simulation study. Ann Biomed Eng. 2007;35:1756-70.

172. Tice BM, Rodríguez B, Eason J, Trayanova N. Mechanistic investigation into the arrhythmogenic role of transmural heterogeneities in regional ischaemia phase 1A. Europace. 2007;9 Suppl 6:vi46-58.

173. Romero L, Trénor B, Alonso JM, Tobón C, Saiz J, Ferrero Jr JM. The relative role of refractoriness and source-sink relationship in reentry generation during simulated acute ischemia. Ann Biomed Eng. 2009;37:1560-71.

174. Heidenreich EA, Ferrero JM, Rodríguez JF. Modeling the human heart under acute ischemia. In: Calvo Lopez B, Peña E, editors. Patient-Specific Comput Model. Volume 5. Netherlands: Dordrecht: Springer; 2012. p. 81-103 [Lecture Notes in Computational Vision and Biomechanics].

175. Rodríguez B, Tice BM, Eason JC, Aguel F, Ferrero Jr JM, Trayanova N. Effect of acute global ischemia on the upper limit of vulnerability: a simulation study. Am J Physiol Heart Circ Physiol. 2004;286:H2078-88.

176. Lazzara R, Scherlag BJ. Electrophysiologic basis for arrhythmias in ischemic heart disease. Am J Cardiol. 1984;53:B1-7.

177. Vigmond E, Vadakkumpadan F, Gurev V, Arevalo H, Deo M, Plank G, et al. Towards predictive modelling of the electrophysiology of the heart. Exp Physiol. 2009;94:563-77.

178. Rantner U, Arevalo HJ, Constantino JL, Efimov IR, Plank G, Trayanova NA. Three-dimensional mechanisms of increased vulnerability to electric shocks in myocardial infarction: altered virtual electrode polarizations and conduction delay in the peri-infarct zone. J Physiol. 2012;590:4537-51.

179. Pop M, Sermesant M, Mansi T, Crystal E, Ghate S, Peyrat JM, et al. Correspondence between simple 3-D MRI-based computer models and in-vivo EP measurements in swine with chronic infarctions. IEEE Trans Biomed Eng. 2011;58:3483-6.

180. Ng J, Jacobson JT, Ng JK, Gordon D, Lee DC, Carr JC, et al. Virtual electrophysiological study in a 3-dimensional cardiac magnetic resonance imaging model of porcine myocardial infarction. J Am Coll Cardiol. 2012;60:423-30.

181. Arevalo H, Plank G, Helm P, Halperin H, Trayanova N. Tachycardia in post-infarction hearts: insights from 3D image-based ventricular models. PLoS One. 2013;8, e68872.

182. Kerckhoffs RCP, Neal ML, Gu Q, Bassingthwaighte JB, Omens JH, McCulloch AD. Coupling of a 3D finite element model of cardiac ventricular mechanics to lumped systems models of the systemic and pulmonic circulation. Ann Biomed Eng. 2007;35:1-18.

183. Kerckhoffs RCP, Campbell SG, Flaim SN, Howard EJ, Sierra-Aguado J, Mulligan L, et al. Multi-scale modeling of excitation-contraction coupling in the normal and failing heart. Annu Int Conf IEEE Eng Med Biol Soc. 2009;2009:4281-2. 
184. Sermesant M, Chabiniok R, Chinchapatnam P, Mansi T, Billet F, Moireau P, et al. Patient-specific electromechanical models of the heart for the prediction of pacing acute effects in CRT: a preliminary clinical validation. Med Image Anal. 2012;16:201-15

185. Vinson CA, Gibson DG, Yettram AL. Analysis of left ventricular behaviour in diastole by means of finite element method. Br Heart J. 1979;41:60-7.

186. Yamaki M, Kubota I, Tomoike H. Simulation of late potentials and arrhythmias by use of a three-dimensional heart model: casuality of peri-infarctional slow conduction in ventricular fibrillation. J Electrocardiol. 1999;32:115-21.

187. Kerfoot E, Lamata P, Niederer S, Hose R, Spaan J, Smith N. Share and enjoy: anatomical models database - generating and sharing cardiovascular model data using web services. Med Biol Eng Comput. 2013;51:1181-90.

\section{Submit your next manuscript to BioMed Central and take full advantage of:}

- Convenient online submission

- Thorough peer review

- No space constraints or color figure charges

- Immediate publication on acceptance

- Inclusion in PubMed, CAS, Scopus and Google Scholar

- Research which is freely available for redistribution 